\title{
Convection-Enhanced Diffusion for Random Flows
}

\author{
Albert Fannjiang ${ }^{1}$ and George Papanicolaou ${ }^{2}$
}

Received March 11, 1996; final April 11, 1997

\begin{abstract}
We analyze the effective diffusivity of a passive scalar in a two-dimensional, steady, incompressible random flow that has mean zero and a stationary stream function. We show that in the limit of small diffusivity or large Peclet number, with convection dominating, there is substantial enhancement of the effective diffusivity. Our analysis is based on some new variational principles for convection diffusion problems and on some facts from continuum percolation theory, some of which are widely believed to be correct but have not been proved yet. We show in detail how the variational principles convert information about the geometry of the level lines of the random stream function into properties of the effective diffusivity and substantiate the result of Isichenko and Kalda that the effective diffusivity behaves like $\varepsilon^{3 / 13}$ when the molecular diffusivity $\varepsilon$ is small, assuming some percolation-theoretic facts. We also analyze the effective diffusivity for a special class of convective flows, random cellular flows, where the facts from percolation theory are well established and their use in the variational principles is more direct than for general random flows.
\end{abstract}

KEY WORDS: Diffusion, convection; random media; percolation.

\section{INTRODUCTION}

The concentration density $\rho$ of a cloud of passive particles diffusing with molecular diffusivity $\varepsilon$ and convecting in an incompressible fluid flow $\mathbf{u}$, with $\nabla \cdot \mathbf{u}=0$, satisfies the convection-diffusion equation

$$
\frac{\partial \rho}{\partial t}(\mathbf{x}, t)+\mathbf{u}(\mathbf{x}) \cdot \nabla \rho(\mathbf{x}, t)=\varepsilon \Delta \rho(\mathbf{x}, t)
$$

\footnotetext{
${ }^{1}$ Department of Mathematics, University of California at Davis, Davis, California 95616; e-mail: fannjian@math.ucdavis.edu.

${ }^{2}$ Department of Mathematics, Stanford University, Stanford, California 94305; e-mail: papanico@math.stanford.edu.
} 


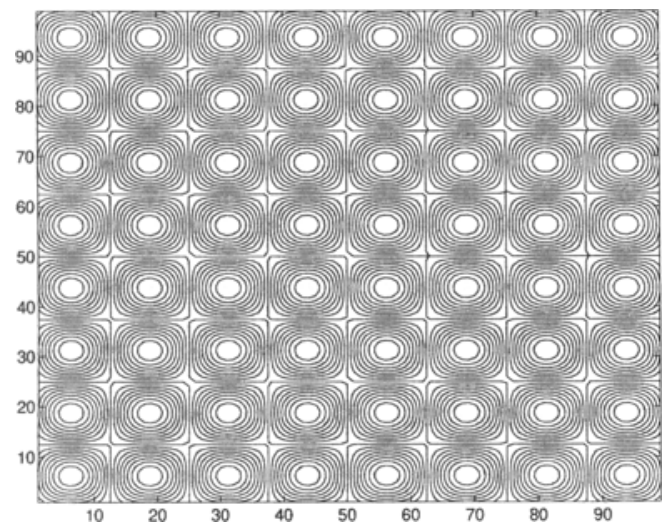

Fig. 1. Periodic cellular flow.

with the initial concentration $\rho(x, 0)=\rho_{0}(\mathbf{x})$ given. The paths of the particles are determined from the stochastic differential equation

$$
d \mathbf{x}(t)=\mathbf{u}(\mathbf{x}(t)) d t+\sqrt{2 \varepsilon} d \mathbf{w}(t)
$$

with $\mathbf{x}(0)=\mathbf{x}_{0}$ given and with $\mathbf{w}(t)$ the standard Brownian motion. In two dimensions, the divergence free condition

$$
\nabla \cdot \mathbf{u}=0
$$

determines a stream function $\psi(\mathbf{x})$ such that

$$
\mathbf{u}(x, y)=\left(-\psi_{y}, \psi_{x}\right)=\nabla^{\perp} \psi(x, y)
$$

Figure 1 shows the streamlines of the periodic cellular flow given by

$$
\psi(x, y)=\sin x \cdot \sin y
$$

while those of perturbed cellular flows look like Figs. 2, 3, with an additive periodic and random perturbation, respectively.

The large scale transport properties of (1.1) are characterized by the effective diffusivity matrix $\sigma_{\varepsilon}$, when the velocity field $\mathbf{u}$ has a repetitive structure, as in the case of a periodic or a stationary random flow with mean zero. It is defined as the asymptotic rate of mean square displacement in the direction $\mathbf{e}$ :

$$
\sigma_{\varepsilon}(\mathbf{e})=\lim _{t \rightarrow \infty} \frac{\left|\mathbf{x}_{\varepsilon}(t) \cdot \mathbf{e}\right|^{2}}{t}
$$




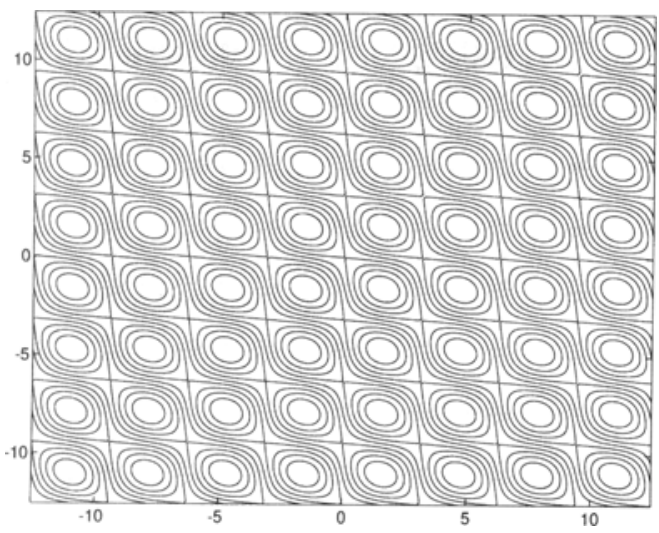

Fig. 2. Cat's-eye flow with $\delta=0.2$.

where the scaled process is

$$
\mathbf{x}_{\varepsilon}=\varepsilon \mathbf{x}\left(\frac{t}{\varepsilon^{2}}\right)
$$

The limit (1.6) exists with probability one for a large class of flows (see, e.g., ref. 11). The quadratic form $\sigma_{\varepsilon}(\mathbf{e})$ can be characterized in other ways as well, as, for example, by the cell problem (2.8) that is discussed in Section 2 . We are interested here in the effective diffusivity of random flows and its behavior as the molecular diffusivity $\varepsilon$ tends to zero. This is the high Peclet number regime that is important for the understanding of transport

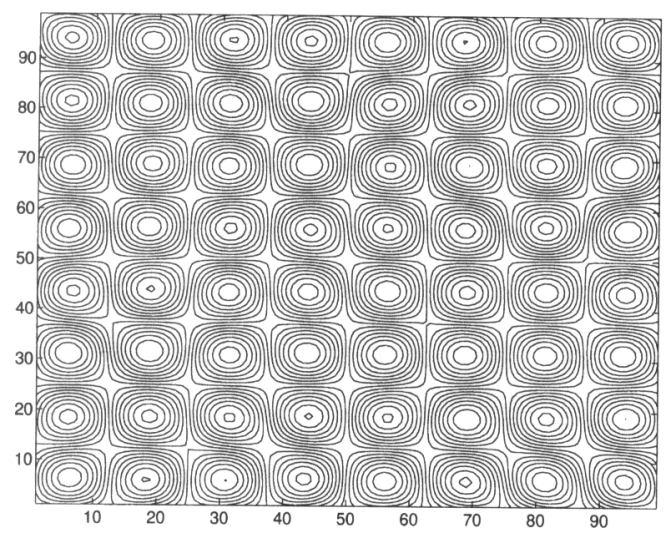

Fig. 3. Cellular flow with random perturbation. 
processes in real turbulent flows where the molecular diffusivity is often quite small. In general, we have the bounds ${ }^{(2,9)}$

$$
c_{1} \varepsilon \leqslant \sigma_{s}(\mathbf{e}) \leqslant \frac{c_{2}}{\varepsilon}
$$

as $\varepsilon \rightarrow 0$, with $c_{1}$ and $c_{2}$ constants, which are not, however, very informative since they do not take into account the geometric structure of the flow. A rigorous and rather full analysis of the large Peclet number regime for two dimensional periodic flows was given in ref. 9 using extensively variational methods that were introduced there. The variational principles were also derived by Cherkaev and Gibiansky in ref. 5 . It was shown in ref. 9 that we have

$$
\sigma_{\varepsilon} \sim c^{*} \varepsilon^{\alpha} \quad \text { as } \quad \varepsilon \rightarrow 0
$$

and fluid flows for which $\alpha=-1$, or $\alpha \in[1 / 2,1]$, with even a logarithm such as

$$
\frac{1}{c} \varepsilon \log \frac{1}{\varepsilon} \leqslant \sigma_{\varepsilon} \leqslant c \varepsilon \log \frac{1}{\varepsilon}, \quad \text { as } \quad \varepsilon \rightarrow 0
$$

can be explicitly identified in two dimensions, in terms of the geometry of the streamlines. The exponent $\alpha$ depends on the critical streamline geometry of the flows. For example, $\alpha=1 / 2$ for the cellular flow (1.5) (see also refs. 6, 20, and 21) and $\alpha=1, \alpha=-1$ for shear flows (see also ref. 7), in the direction perpendicular to the flow and along the flow, respectively. The constant $c^{*}$ can be calculated explicitly in several cases. Our analysis in ref. 9 makes precise the relation between streamline geometry and the large Peclet number behavior of the effective diffusivity $\sigma_{\varepsilon}$ for two dimensional steady, incompressible, periodic flows.

In this paper we extend the analysis and the use of variational methods to random flows. One important difference in the random case is that the cell problem for the effective diffusivity $(2.8)$ is over the whole space $R^{2}$, rather than a periodic domain, and it is difficult to work with even though the mean field conditions (2.10) are convenient. It is handled through an infinite volume limit with appropriate boundary conditions and the more flexible these boundary conditions are, the easier it is to work with them. In Section 2 we explain how the effective diffusivity can be calculated with convenient boundary conditions, in an infinite volume limit.

The direct and dual variational principles for the infinite volume limit are also presented in Section 2. The direct and dual minimum principles are used to obtain upper and lower bounds for the effective diffusivity. This is the way they were used in the periodic case. ${ }^{(9)}$ But it is possible in 
principle, and sometimes desirable, to stay within the framework of the direct principles, without going to the duals, to estimate $\sigma_{\varepsilon}$ from above and below. We demonstrate this possibility for the periodic cellular flow in the Appendix. This is important when the effective diffusivity is not symmetric and the utility of the dual principles is restricted.

The random flows considered in this paper are perturbations of the periodic cellular flow (1.5). This flow is degenerate because all its hyperbolic stagnation points, and thus all separatrices, are on the same level set of the stream function $\psi$, that is, the set of points where $\psi=\sin x \cdot \sin y=0$ is a periodic array of separatrices with the streamlines confined within each cell. This cellular flow structure is not stable under perturbations. Distinctive flows will emerge when perturbations $\delta \psi_{1}$ are introduced

$$
\psi(x, y)=\sin x \cdot \sin y+\delta \psi_{1}(x, y)
$$

The perturbations destroy the regular lattice of separatrices by redistributing the hyperbolic stagnation points to different level sets of $\psi$ so that streamlines are not confined inside the cells and those nearby the boundaries of cells may reconnect in various ways depending on the properties of $\psi_{1}$. However, the elliptic stagnation points at the center of the cells are stable to perturbations and survive along with the neighboring closed streamlines, the vortex islands. Typically, the phase plane divides into the complicated, reconnected (into closed loops or open channels) streamlines and into islands of vortices.

When, for example, $\psi_{1}$ is doubly periodic in $x, y$ such as $\psi_{1}(x, y)=$ $\cos x \cdot \cos y$, Fig. 2, there is interaction between $\psi_{1}$ and $\sin x \cdot \sin y$ and reconnection of the streamlines creates periodic arrays of open channels. Note that the degeneracy remains because the hyperbolic stagnation points split between the level sets of $\psi=\delta$ and $\psi=-\delta$, instead of being mostly different from each other. When $\psi_{1}$ is quasi-periodic, such as $\psi_{1}(x, y)=$ $\cos (p x+q y) \cdot \cos (-q x+p y)$ with $p^{2}+q^{2}=1$ and $p, q$ rationally independent, then the flow can have a rather irregular channel structure. Similar structures occur with perturbations by constant drifts: $\psi_{1}(x, y)=$ $c_{1} x+c_{2} y$. Depending on the rationality or irationality of $c_{1} / c_{2}$, there are periodic or quasi-periodic channels.

Time dependent perturbations, such as $\psi_{1}(x, y, t)=\phi(x, y)+\sin t$, with $\phi$ periodic in $x$ and $y$, can create quite complicated flow structures for the Lagrangian trajectories and lead to chaotic dynamics. This does not occur in steady periodic flows in two dimensions. But stochastic streamlines will arise for flows with stream function (1.11) when the perturbation $\psi_{1}(x, y)$ is a random function. In Section 3 we consider randomly perturbed stream functions which have roughly the following features: 
- The hyperbolic stagnation points are preserved, are generic and their elevations are independent, identically distributed random variables of size $\delta$.

- The zero level set $\psi=0$ stays close to the lattice $(\pi Z)^{2}$ of unperturbed separatrices and is not degenerate so that nearby level sets and their reconnections are essentially determined, in a way explained in Section 3, by the elevations of the hyperbolic stagnation points and the nearby streamlines.

In this case, there is no systematic or resonant reconnection, as when $\psi_{1}=\cos x \cdot \cos y$, and instead of open channels arbitrarily long streamlines emerge, which are like the perimeters of connected clusters in two dimensional bond percolation at critical probability. The rest of the streamlines reconnect randomly into closed loops of different lengths. The distribution of lengths is similar to that of cluster sizes in near critical bond percolation.

This qualitative description of the geometry of the level lines of randomly perturbed stream functions has not been rigorously established. We also do not have a mathematical analysis for the critical exponents of perimeter lengths of the streamlines. However, it is reasonable to use the results from lattice bond percolation theory in two dimensions, some of which have been proved and the rest are widely believed to be true, as we explain further in Section 3.1.2. This is the main reason that we only consider stream functions of the form (1.11) that are small perturbations of the periodic cellular stream function.

In refs. 14, 15, Isichenko, Kalda and Gruznov used a scaling argument in the Lagrangian description (1.2) based on the broken coherence hypothesis and concluded that

$$
c_{1} \varepsilon^{3 / 13} \leqslant \sigma_{\varepsilon} \leqslant c_{2} \varepsilon^{3 / 13}, \quad \text { as } \quad \varepsilon \rightarrow 0
$$

for randomly perturbed cellular flows described above. The broken coherence hypothesis is basically a separation of scales hypothesis and postulates that the effect of long streamlines on the particle trajectories is independent of that of short streamlines, on long times scales, so that the contribution to the effective diffusivity $\sigma_{\varepsilon}$ over different scales can be simply summed up. A different scaling argument, based on the variational principles, is given in Section 3.2.

In breaking the degeneracy of the periodic stream function $\sin x \sin y$ with random perturbations, we are interested in the universality, if any, of the power law (1.12) for the effective diffusivity of convection dominated turbulent convection-diffusion. We note, for example, that the stream functions (1.11) that we are considering here are different from those studied by 
Alexander and Molchanov; ${ }^{(1)}$ theirs are not small random perturbations of a cellular stream function. We do not know how to extend our analysis to the stream functions of Alexander and Molchanov. We do not expect that the convection dominated behavior of the effective diffusivity will obey the power law (1.12) for the stream functions of Alexander and Molchanov.

In applying the variational methods to random convection-diffusion our aim is to get sharp estimates for the effective diffusivity $\sigma_{\varepsilon}$. For this we need good trial functions and the evaluation of the variational principles for them. The evaluation is an essential part of the method and is not routine because we are dealing with nonlocal variational principles. The nonlocality is the price we pay for being able to use a variational principle for a nonsymmetric problem like convection-diffusion. As noted above, the structure of the large scale streamline geometry is not yet analyzed mathematically for the randomly perturbed stream functions so there is no proof that the trial functions we introduce and use exist. However, their existence is entirely dependent on the geometry of the random streamlines and is quite clear if that is well understood. In this paper, we focus on the use of the variational principles to translate in a transparent way geometric streamline information to the large Peclet number behavior of the effective diffusivity.

In Section 4, we consider convection-diffusion in flows that have a multiplicative random perturbation of the form

$$
\psi(x, y)=\psi_{1}(x, y) \sin x \cdot \sin y
$$

where $\psi_{1}(x, y)$ is the characteristic function of the random checkerboard configuration. This artificial flow maintains the cellular structure of (1.5) while suppressing randomly from cell to cell the motion inside the cells. The random geometry of the remaining cells is known precisely from lattice site percolation theory and the construction of trial functions is now fully justified. This is a relatively simple, but somewhat artificial, example of a random flow where all steps in the analysis based on the variational principles can be justified.

\section{THE EFFECTIVE DIFFUSIVITY}

\subsection{Long Time, Long Distance Behavior of the Density}

Using the skew-symmetric stream matrix

$$
\Psi(\mathbf{x})=\left(\begin{array}{cc}
0 & -\psi(\mathbf{x}) \\
\psi(\mathbf{x}) & 0
\end{array}\right)
$$


the convection-diffusion equation (1.1) can be written in divergence form

$$
\frac{\partial \rho(\mathbf{x}, t)}{\partial t}=\nabla \cdot(\varepsilon+\Psi(\mathbf{x})) \nabla \rho(\mathbf{x}, t)
$$

with initial concentration $\rho(\mathbf{x}, 0)=\rho_{0}(\mathbf{x})$. In homogenization ${ }^{(3,16,19)}$ we look for the long time large distance behavior of solutions of (2.2). This can be done with a large parameter $n>0$ by replacing $t$ by $n^{2} t$ and $\mathbf{x}$ by $n \mathbf{x}$ in (2.2). We then have

$$
\frac{\partial \rho_{n}(\mathbf{x}, t)}{\partial t}=\nabla \cdot(\varepsilon+\Psi(n \mathbf{x})) \nabla \rho_{n}(\mathbf{x}, t)
$$

where $\rho_{n}(\mathbf{x}, t)=\rho\left(n \mathbf{x}, n^{2} t\right)$, and $\Psi_{n}(\mathbf{x})=\Psi(n \mathbf{x}), \mathbf{x}=(x, y)$. We assume that the initial data do not depend on $n$

$$
\rho_{n}(\mathbf{x}, 0)=\rho_{0}(\mathbf{x})
$$

which is equivalent to assuming that they are slowly varying in the unscaled variables.

For a random stream function $\psi(\mathbf{x})$ that is uniformly bounded, or even square integrable

$$
\left\langle|\psi|^{2}(\mathbf{x}, \cdot)\right\rangle<\infty
$$

where $\langle\cdot\rangle$ stands for the ensemble average, it is shown in refs. $3,16,19$, and 10 that the solution of $(2.3), \rho_{n}(\mathbf{x}, t)$, converges to $\bar{\rho}(\mathbf{x}, t)$, the solution of an equation with constant coefficients

$$
\begin{aligned}
\frac{\partial \bar{\rho}}{\partial t} & =\nabla \cdot \frac{1}{2}\left(\sigma_{\varepsilon}+\sigma_{\varepsilon}^{+}\right) \nabla \bar{\rho} \\
\bar{\rho}(\mathbf{x}, 0) & =\rho_{0}(\mathbf{x})
\end{aligned}
$$

where $\sigma_{\varepsilon}$ is the effective diffusivity and $\sigma_{\varepsilon}^{+}$its transpose. The convergence is in $L^{2}$

$$
\sup _{0 \leqslant t \leqslant t_{0}} \frac{1}{|\mathcal{O}|} \int_{\mathscr{Q}} d \mathbf{x}\left|\rho_{n}(\mathbf{x}, t)-\bar{\rho}(\mathbf{x}, t)\right|^{2} \rightarrow 0
$$

as $n \rightarrow \infty$, for any $t_{0}>0$ and for almost all realizations of the random flow. 
The effective diffusivity $\sigma_{\varepsilon}$ is in general a nonsymmetric matrix but has a positive definite symmetric part and is determined by a random cell problem. This means that we must find for each unit vector e a random function $\chi=\chi(\mathbf{x} ; \mathbf{e})$ which is the unique (up to a constant) solution of

$$
\nabla \cdot[(\varepsilon \mathbf{I}+\mathbf{H})(\nabla \chi+\mathbf{e})]=0
$$

with $\nabla \chi$ a stationary, mean zero random field. The effective diffusivity matrix, denoted by $\sigma_{\bar{\varepsilon}}$, is given by

$$
\sigma_{\varepsilon}(\mathbf{e})=\sigma_{\varepsilon} \mathbf{e} \cdot \mathbf{e}=\sigma_{\varepsilon}(\mathbf{e})=\varepsilon\langle(\nabla \chi+\mathbf{e}) \cdot(\nabla \chi+\mathbf{e})\rangle=\varepsilon+\varepsilon\langle\nabla \chi \cdot \nabla \chi\rangle
$$

We see, therefore, that the small diffusion limit $(\varepsilon \rightarrow 0)$ of the effective diffusivity $\sigma_{\varepsilon}$ amounts to the analysis of the singularly perturbed, random diffusion equation (2.8) on the entire space. Note also that convection always enhances diffusion since $\sigma_{\varepsilon}(\mathbf{e}) \geqslant \varepsilon$.

The fact that the cell problem (2.8) determines the effective diffusivity can be understood physically as follows. We state this for a problem in $R^{d}$ even though we deal with $d=2$ only here. Let $\left\{\mathbf{e}_{j}\right\}$ be a basis of orthogonal unit vectors in $\mathbf{R}^{d}$, let $\chi_{j}$ be the solution of the cell problem (2.8) and let

$$
\mathbf{E}_{j}=\nabla \chi_{j}+\mathbf{e}_{j}
$$

Then $\mathbf{E}_{\boldsymbol{j}}$ is the field intensity, the concentration or temperature gradient, and

$$
\mathbf{D}_{j}=(\varepsilon I+\Psi) \mathbf{E}_{j}
$$

is the flux. Since $\Psi$ is skew symmetric, the intensity-flux relation is not the usual Fourier law but resembles that of a Hall medium. From (2.8) and (2.10) we see that

$$
\nabla \times \mathbf{E}_{j}=0, \quad \nabla \cdot \mathbf{D}_{j}=0, \quad\left\langle\mathbf{E}_{j}\right\rangle=\mathbf{e}_{j}
$$

and

$$
\sigma_{\varepsilon}\left\langle\mathbf{E}_{j}\right\rangle=\left\langle\mathbf{D}_{j}\right\rangle
$$

Relation (2.11) is the linear constitutive law relating intensity and flux. Equations (2.12) tell us that $\mathbf{E}_{j}$ is a gradient, that there are no sources or sinks and that the mean or imposed intensity is a unit vector in the direction $\mathbf{e}_{j}$. The effective diffusivity $\sigma_{\varepsilon}$, is defined by (2.13), which is the linear 
constitutive law relating mean intensity and mean flux. It is in general a nonsymmetric matrix given by

$$
\begin{aligned}
\sigma_{\varepsilon} \mathbf{e}_{i} \cdot \mathbf{e}_{j}=\sigma_{\varepsilon}\left(\mathbf{e}_{i}, \mathbf{e}_{j}\right) & =\left\langle\mathbf{D}_{i} \cdot \mathbf{e}_{j}\right\rangle \\
& =\left\langle(\varepsilon I+\Psi) \mathbf{E}_{i} \cdot \mathbf{E}_{j}\right\rangle
\end{aligned}
$$

It is shown in ref. 10 that $\sigma_{\varepsilon}$ is symmetric if the probability distribution of the streamfunctions is invariant under the transform

$$
\psi(\mathbf{x}) \rightarrow-\psi(\mathbf{x})
$$

The sign symmetry condition $(2.15)$ is satisfied for the random flows considered in Section 3 and 4. This makes it easier to apply the dual variational principle to obtain lower bounds for $\sigma_{\varepsilon}$.

\subsection{Infinite Volume Limit for the Effective Diffusivity}

The effective diffusivity is characterized by the random cell problem (2.8) in a precise way. In the periodic case, it reduces to a standard elliptic equation in a period cell and admits nonlocal variational principles and their duals, defined over a period cell, which in turn can be used to obtain sharp estimates on the effective diffusivity in the small diffusion regime ( $\varepsilon \ll 1$, see ref. 9 for details). But in the random case, the use of $(2.8)$ over the whole space is rather limited. To analyze this problem we have to approach it via an infinite volume limit of periodic cell problems with suitable, periodic boundary conditions. These periodized, finite volume cell problems are as follows.

Let us assume for simplicity that $\left\{\mathbf{e}_{i}\right\}$ is the set of the orthonormal eigenvectors of $1 / 2\left(\sigma_{\varepsilon}+\sigma_{\varepsilon}^{\dagger}\right)$ and let $\tilde{\psi}_{n}$ be the periodic stream function defined on $[-1,1]^{2}$ such that

$$
\begin{aligned}
& \tilde{\psi}_{n}\left(-x_{1}, x_{2}\right)=\tilde{\psi}_{n}\left(x_{1}, x_{2}\right) \\
& \tilde{\psi}_{n}\left(x_{1},-x_{2}\right)=\tilde{\psi}_{n}\left(x_{1}, x_{2}\right)
\end{aligned}
$$

Clearly, $\tilde{\psi}_{n}(-\mathbf{x})=\tilde{\psi}_{n}(\mathbf{x})$. Let $\tilde{\sigma}_{\varepsilon, n}$ be the effective diffusivity of the periodized flow constructed above. This depends on the realization of the random medium because the average $\langle\cdot\rangle$ in $(2.9)$ is now a volume average overthe period cell. A corollary of homogenization theory is the convergence of the effective diffusivities of the periodized cell problems. That is, if $\left|\widetilde{\psi}_{n}\right|$ is uniformly bounded and $\left\{\mathbf{e}_{i}\right\}$ the set of eigenvectors of the symmetric part of $\sigma_{\varepsilon}$ then

$$
\lim _{n \rightarrow \infty} \tilde{\sigma}_{\varepsilon, n}\left(\mathbf{e}_{i}, \mathbf{e}_{j}\right)=\sigma_{\varepsilon}\left(\mathbf{e}_{i}, \mathbf{e}_{j}\right), \quad i, j=1,2
$$


with probability one with respect to the random medium. In the proof of this result we can use the variational methods developed for periodic flows in ref. 9 which we will now summarizes in ce we need them for the small $\varepsilon$ analysis.

\subsection{Variational Principles}

We summarize here the facts about variational principles for the symmetric part of $\tilde{\sigma}_{\varepsilon, n}$ (see ref. 9 for details) which will be needed in this paper.

Let $\mathscr{H}_{g}\left([-1,1]^{2}\right), \mathscr{H}_{c}\left([-1,1]^{2}\right)$ be the Hilbert spaces defined by

$$
\begin{aligned}
& \mathscr{H}_{g}\left([-1,1]^{2}\right)=\left\{\mathbf{F} \text { periodic in }[-1,1]^{2} \mid \nabla \times \mathbf{F}=0, \int_{[-1,1]^{2}} d \mathbf{x} \mathbf{F}=0\right\} \\
& \mathscr{H}_{c}\left([-1,1]^{2}\right)=\left\{\mathbf{G} \text { periodic in }[-1,1]^{2} \mid \nabla \cdot \mathbf{G}=0, \int_{[-1,1]^{2}} d \mathbf{x} \mathbf{G}=0\right\}
\end{aligned}
$$

The subscript $g$ stands for gradient fields and the subscript $c$ for curl fields, respectively. We have first the direct min-max variational principle for $\tilde{\sigma}_{\varepsilon, n}\left(\mathbf{e}_{i}\right)$, as in Appendix A.2 of ref. 9

$$
\begin{aligned}
\tilde{\sigma}_{\varepsilon, n}\left(\mathbf{e}_{i}\right)= & \inf _{\mathbf{F} \in \mathbf{e}_{i}+\mathscr{H}_{g}\left[[-1,1]^{2}\right)} \sup _{\mathbf{F}^{\prime} \in \mathscr{H}_{g}\left([-1,1]^{2}\right)} \frac{1}{2^{2}} \int_{[-1,1]^{2}} d \mathbf{x} \\
& \times\left\{\varepsilon \mathbf{F} \cdot \mathbf{F}-2 \widetilde{\Psi}_{n} \mathbf{F} \cdot \mathbf{F}^{\prime}-\varepsilon \mathbf{F}^{\prime} \cdot \mathbf{F}^{\prime}\right\}
\end{aligned}
$$

Eliminating the supremumin (2.20) by solving the corresponding Euler equation

$$
\begin{aligned}
\nabla \cdot \mathbf{F}^{\prime}+\nabla \cdot \widetilde{\Psi}_{n} \mathbf{F} & =0 \\
\frac{1}{2^{2}} \int_{[-1,1]^{2}} d \mathbf{x} \mathbf{F}^{\prime} & =0
\end{aligned}
$$

we obtain the nonlocal, direct minimum principle $\tilde{\sigma}_{\varepsilon, n}$ :

$$
\tilde{\sigma}_{\varepsilon, n}\left(\mathbf{e}_{i}\right)=\inf _{\mathbf{F} \in \mathbf{e}_{i}+\mathscr{H}_{g}\left([-1,1]^{2}\right)} \frac{1}{2^{2}} \int_{[-1,1]^{2}} d \mathbf{x}\left\{\varepsilon \mathbf{F} \cdot \mathbf{F}+\frac{1}{\varepsilon} \Gamma \tilde{\Psi}_{n} \mathbf{F} \cdot \Gamma \tilde{\Psi}_{n} \mathbf{F}\right\}
$$


where

$$
\Gamma=\nabla \Delta^{-1} \nabla .
$$

is the projection onto the zero mean gradient fields.

One may also get a maximum principle by eliminating the infimum

$$
\begin{aligned}
\tilde{\sigma}_{\varepsilon, n}\left(\mathbf{e}_{i}\right)= & \sup _{\mathbf{F}^{\prime} \in \mathscr{H}_{g}\left([-1,1]^{2}\right)} \frac{1}{2^{2}} \int_{[-1,1]^{2}} d \mathbf{x} \\
& \times\left\{-\varepsilon \mathbf{F}^{\prime} \cdot \mathbf{F}^{\prime}-\frac{1}{\varepsilon} \Gamma \tilde{\Psi}_{n} \mathbf{F}^{\prime} \cdot \Gamma \tilde{\Psi}_{n} \mathbf{F}^{\prime}+2(I-\Gamma) \tilde{\Psi}_{n} \mathbf{F}^{\prime} \mathbf{e}_{i}+\varepsilon\right\}
\end{aligned}
$$

This maximum principle is not very useful in obtaining lower bounds for $\tilde{\sigma}_{\varepsilon, n}\left(\mathbf{e}_{i}\right)$ unless we can evaluate the nonlocal term sufficiently accurately, which we can for periodic laminar flows. We demonstrate this possibility in the Appendix.

In general, we do need the dual minimum principles (Appendix A.1 of ref. 9) to get sharp lower bounds. They have the form

$$
\begin{aligned}
\tilde{\sigma}_{\varepsilon, n}^{-1}\left(\mathbf{e}_{i}\right)= & \inf _{\mathbf{G} \in \mathbf{e}_{i}+\mathscr{H}_{c}\left([-1,1]^{2}\right)} \sup _{\mathbf{G}^{\prime} \in \mathscr{H}_{c}\left([-1,1]^{2}\right)} \frac{1}{2^{2}} \int_{[-1,1]^{2}} d \mathbf{x} \frac{1}{\varepsilon} \frac{1}{1+\frac{1}{\varepsilon^{2}} \tilde{\psi}_{n}^{2}} \\
& \times\left\{\mathbf{G} \cdot \mathbf{G}+\frac{2}{\varepsilon} \widetilde{\Psi}_{n} \mathbf{G} \cdot \mathbf{G}^{\prime}-\mathbf{G}^{\prime} \cdot \mathbf{G}^{\prime}\right\}
\end{aligned}
$$

Eliminating the supremum, we obtain the nonlocal dual minimum principle for the periodized effective resistivity

$\tilde{\sigma}_{\varepsilon, n}^{-1}\left(\mathbf{e}_{i}\right)=\inf _{\mathbf{G} \in \mathbf{e}_{i}+\mathscr{H}_{c}\left([-1,1]^{2}\right)} \frac{1}{2^{2}} \int_{[-1,1]^{2}} d \mathbf{x} \frac{1}{\varepsilon} \frac{1}{1+\frac{1}{\varepsilon^{2}} \tilde{\psi}_{n}^{2}}\left\{\mathbf{G} \cdot \mathbf{G}+\mathbf{G}^{\prime} \cdot \mathbf{G}^{\prime}\right\}$

where the periodic field $\mathbf{G}^{\prime}$ satisfies

$$
\begin{gathered}
\nabla \times\left[\frac{1}{1+\frac{1}{\varepsilon^{2}} \tilde{\psi}_{n}^{2}} \mathbf{G}^{\prime}\right]=\nabla \times\left[\frac{1}{1+\frac{1}{\varepsilon^{2}} \widetilde{\psi}_{n}^{2} \frac{1}{\varepsilon}} \widetilde{\Psi}_{n} \mathbf{G}\right] \\
\int_{[-1,1]^{d}} d \mathbf{x} \mathbf{G}^{\prime}=0
\end{gathered}
$$


Note that because of the symmetries of the extended stream function $\tilde{\psi}_{n}$, we can restrict the trial fields $\mathbf{F}=\nabla f, \mathbf{G}=\nabla^{\perp} g$ for (2.23), (2.27), respectively, to ones with the symmetry

$$
\begin{aligned}
& \left.f\right|_{x_{i}=-1}=\left.2 f\right|_{x_{i}=0}-\left.f\right|_{x_{i}=1},\left.\quad f\right|_{x_{j}=-1}=\left.f\right|_{x_{j}=1}, \quad \forall j \neq i \\
& \left.g\right|_{x_{j}=-1}=\left.2 g\right|_{x_{j}=0}-\left.g\right|_{x_{j}=1},\left.\quad g\right|_{x_{i}=-1}=\left.g\right|_{x_{i}=1}, \quad \forall j \neq i
\end{aligned}
$$

and such that

$$
\begin{gathered}
\frac{1}{4} \int_{[-1,1]^{2}} d \mathbf{x} \mathbf{F}=\mathbf{e}_{i}, \quad \mathbf{F} \text { is periodic in }[-1,1]^{2} \\
\frac{1}{4} \int_{[-1,1]^{2}} d \mathbf{x} \mathbf{G}=(-1)^{i+1} \mathbf{e}_{i}, \quad \mathbf{G} \text { is periodic in }[-1,1]^{2}
\end{gathered}
$$

The symmetry allows us to reduce further the construction of the trial functions to $[0,1]^{2}$ and satisfying

$$
\begin{aligned}
& {[f]_{x_{i}=0}^{x_{i}=1}=\left.f\right|_{x_{i}=1}-\left.f\right|_{x_{i}=0}=1, \quad 0 \leqslant x_{j} \leqslant 1, \quad \forall j \neq i} \\
& {[g]_{x_{j}=0}^{x_{j}=1}=\left.g\right|_{x_{j}=1}-\left.g\right|_{x_{j}=0}=1, \quad 0 \leqslant x_{i} \leqslant 1, \quad \forall j \neq i}
\end{aligned}
$$

We do not need to specify conditions in the direction orthogonal to $\mathrm{e}_{\mathrm{i}}$ since the $\left\{\mathbf{e}_{\mathrm{i}}\right\}$ are assumed to be the eigenvectors of the symmetric part of $\sigma_{\varepsilon}$ so that discrepancies in the boundary data in the orthogonal direction do not affect $\sigma_{\varepsilon, n}\left(\mathbf{e}_{\mathbf{i}}\right)$ in the limit $n \rightarrow \infty$.

\section{RANDOM PERTURBATIONS OF PERIODIC CELLULAR FLOWS}

In this section we attempt to study as rigorously as possible the universality question of the scaling laws for the effective diffusivity and the transport mechanism of the fractal boundary layers arising in random flows whose stream functions are small random perturbations of cellular ones. The variational analysis presented here makes a precise connection between the scaling laws for the effective diffusivity and the ones for the streamline geometry (statistical topography) by using "good" trial functions. Therefore, if there is universality for the former, it is directly related to that of the latter. By changing the probability density of the heights of the saddle points of the perturbed cellular stream function, our analysis 
shows that there is a whole range of scaling exponents in $(0,1 / 2)$ for the effective diffusivity. Among them, $\alpha=3 / 13$ may be considered as a typical one because the height density is bounded above and below over a finite interval in this case.

When the regular lattice of separatrices in the periodic stream function $\sin x \sin y$ is destroyed by random perturbations, it gives rise to closed streamlines whose perimeters vary on many length scales. The molecular diffusivity selects a specific scale that determines the fractal boundary layer for transport.

\subsection{Statistical Topography}

3.1.1. The Random Stream Functions. In this section we first follow ${ }^{(14)}$ in describing the class of randomly perturbed cellular flow stream functions $\psi(\mathbf{x}), \mathbf{x}=(x, y) \in R^{2}$, that we will consider. Then we apply the variational methods of Section 2 to study the behavior of $\sigma_{\varepsilon}$ as $\varepsilon \rightarrow 0$. The random stream functions are sign symmetric, that is, $\psi(x, y)$ is statistically equivalent to $-\psi(x, y)$. Consequently, the effective diffusivities $\sigma_{\varepsilon}$ are symmetric and we assume that they have $\pi / 2$ rotational symmetry.

The level sets of $\sin x \cdot \sin y$ constitute the two dimensional periodic cellular flow. ${ }^{(9)}$ All streamlines are closed and their lengths are uniformly bounded, except for one critical level set which is defined by $\sin x$. $\sin y=0$. This critical level set is an infinite periodic network of heteroclinic orbits (i.e. separatrices) which are unstable under perturbations. The instability comes from all the saddle points having the same elevation.

Now we add a random perturbation $\psi_{1}(x, y)$ to $\sin x \cdot \sin y$ and consider the stream function

$$
\psi(x, y)=\sin x \cdot \sin y+\delta \psi_{1}(x, y)
$$

where $\delta$ is sufficiently small and $\psi_{1}(x, y)$ is $C^{1}$-smooth, uniformly bounded, sign symmetric, statistically invariant under lattice translations and rotations, and with sufficiently fast decaying correlation functions such that:

(A) The vortex islands of $\sin x \cdot \sin y$ are essentially preserved near the center of the cells so that the perturbed $\psi$ changes sign when going from one such region to an adjacent one.

(B) The saddle points, i.e. the hyperbolic stagnation points, are preserved and their elevations are independent, identically distributed random variables with absolute value less than $\delta$.

(C) The locations of the elliptic and hyperbolic stagnation points are essentially those of the square lattice $((\pi / 2) Z)^{2}$. 
(D) The zero level set $\psi=0$ always stays close to the $(\pi Z)^{2}$ lattice of unperturbed separatrices and the function $\psi$ is not degenerate so that near by level sets and their reconnections are essentially determined by the elevations of the saddle points they pass by, as explained below.

Condition (A) is a simple consequence of the KAM theory in view of the Hamiltonian structure of two dimensional incompressible flows. The first half of (B) is implied by the stability of hyperbolic stagnation points. The distribution and correlation of the saddle point elevations are completely determined by $\psi_{1}$ since they are at the same elevation before perturbation. Condition (C) can be relaxed and together with (B) and (D) it enables us to stay as close to the lattice bond percolation models as possible.

In short, the saddle points of the perturbed stream function maintain the lattice structures, i.e. they are the sites of the lattice $(\pi Z)^{2}$, and the saddle point elevations are i.i.d. random variables.

Let us give an example in which all of the preceding conditions (A)-(D) are satisfied. Take any i.i.d. random variables on the lattice sites of $(\pi Z)^{2}$ distributed with a density compactly supported in $[-1,1]$ and symmetric with respect to zero. To build the perturbation function $\psi_{1}(x, y)$, we first interpolate on the lattice bonds such that the derivatives at sites are zero. This can be done by cubic polynomials and the result is a $C^{1}$ function defined on the two dimensional lattice with zero derivatives at lattice sites. Next, we extend this function to the entire plane by interpolating it square by square. To ensure the $C^{1}$-ness across the lattice bonds, we demand that the extended function has zero normal derivatives on the lattice bonds. Now the construction redues to the existence of a collection of $C^{1}$ functions on a square with cubic polynomials as Dirichlet data and zero Neumann data which is clear. Condition (A) holds for sufficiently small $\delta$ because of KAM theorem. Since the lattice sites are also the stagnation points of the perturbation $\psi_{1}$ and the function $\sin x \sin y$, and since at those sites the former is i.i.d. random variables and the latter is zero, Condition (B) is satisfied. Condition (C) is really a consequence of (A) and (B). The zero level $\psi=0$ stays close to the lattice because of small perturbation. The function is no longer degenerate near the zero level $\psi=0$ since the elevations of saddle points are i.i.d. Thus (D) is satisfied too.

As a consequence of the random saddle point elevations the infinite periodic network of separatrices, together with the web of its $\delta$-neighborhood, is essentially destroyed. Since $\psi_{1}$ is sign symmetric, $\psi(x, y)=0$ is still the critical level set. It has infinite length since $\{\psi>0\}$ and $\{\psi<0\}$ are statistically equivalent and unbounded, and it must extend to infinity in an irregular fashion because both $\{\psi>0\}$ and $\{\psi<0\}$ are stationary 
random sets with $\pi / 2$-rotational symmetry. The rest of the level sets are disconnected and each piece has finite length; there cannot be two infinite isotropic level sets. Along with the regularity of the streamlines, the distribution of their lengths is also changed to that of the near critical power laws of bond percolation theory.

Note that the perturbed $\psi$ is stationary in the probability space which is the product of the period cell for the unperturbed stream function and the ensemble of the random perturbations, which have rapidly decaying correlations.

\subsubsection{Connection with the Lattice Bond Percolation}

Models. The statistical topography of the streamlines is a collection of continuum percolation models which may be analyzed by mapping them to lattice bond percolation models. The idea of mapping continuum to lattice percolation models is presented in $\operatorname{Ziman}^{(25)}$ and was developed further by Weinrib. $^{(23)}$

We first identify the local minima of $\psi(x, y)$ as sites. Two neighboring sites are connected by steepest descent lines passing through saddle points. We identify the steepest descent lines as bonds. They form essentially the $\pi / 4$-tilted square lattice $(\sqrt{2} \pi Z)^{2}$. To determine a given level set $\psi=h$, for $|h|<\delta$, we shall construct a model of independent bond lattice percolation with bond occupation probability $p(h)$ : a bond is said to be occupied if the prescribed elevation $h$ is higher than the saddle point elevation $\psi_{s}$ on the bond. When this occurs, the neighboring sites are called connected. Clearly, the backbone of $\{\psi \leqslant h\}$ corresponds to the random cluster of occupied bonds and the level set $\psi(x, y)=h$ is essentially the perimeter of the unperturbed cells of connected sites (Fig. 4).

The occupation probability $p(h)$ is

$$
p(h)=\int_{-\infty}^{h} P\left(\psi_{s}\right) d \psi_{s}, \quad \text { for }
$$

where $P\left(\psi_{s}\right)$ is the probability density of the saddle point elevation, i.e. the one point distribution of $\psi$ at the saddle points, assumed to have support in $[-\delta, \delta]$. The critical bond percolation probability $p_{c}=\frac{1}{2}$ corresponds to the critical elevation $h_{c}=0$. The level set $\psi(x, y)=h_{c}$ corresponds to the infinite perimeter of the unique infinite bond percolation cluster at $p=p_{c}$. Thus we may view the statistical topography of $|\psi| \leqslant h$ as a family of near critical bond percolation models depending on $h$. Not surprisingly, the near zero level sets completely determine the asymptotics of $\sigma_{\varepsilon}$ for small $\varepsilon$.

In general, we may take the saddle point elevation density to have the form

$$
P\left(\psi_{s}\right) \sim\left|\psi_{s}\right|^{\psi}, \quad \text { for } \quad\left|\psi_{s}\right| \ll 1
$$


with $\gamma>-1$. Negative $\gamma$ means concentration of saddle points at the zero level, with the periodic cellular flow being the extreme, while positive $\gamma$ means scarcity, the cat's-eye flow with open channels being the extreme (Fig. 2). The case $\gamma=0$ is the one on which we focus our analysis and may be considered as typical.

The near critical behavior of bond percolation clusters in two-dimension is known very well ${ }^{(18)}$ (but little is proved rigorously). When $h \neq h_{c}$, all the clusters are of finite size in the sense that clusters whose size is larger than the correlation length

$$
\xi(h) \sim h^{-\nu}, \quad \text { for } \quad|h|<<\delta, \quad v=\frac{4}{3}
$$

are exponentially scarce. We may think of $\xi(h)$ as the typical diameter of the level set $\psi(x, y)=h$. There is another nontrivial hull exponent, $d_{H}$, denoting the fractal dimension of a large connected clusters of occupied bonds. It is known ${ }^{(22)}$ (and numerically confirmed ${ }^{(24)}$ but not proved rigorously) that

$$
d_{H}=1+1 / v=\frac{7}{4}
$$

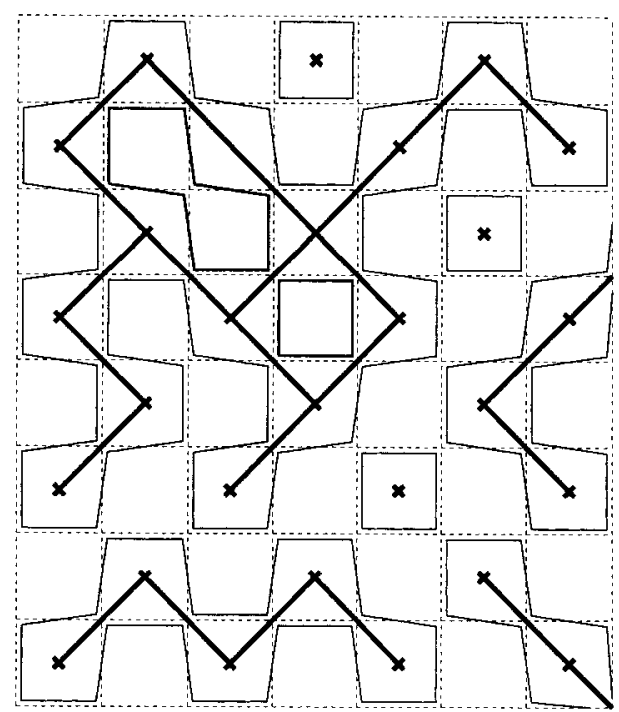

Fig. 4. Connection with bond percolation models: Dashed lines are the separatrices of the original lattice $(\pi Z)^{2}$ of the per iodic cellular flow. Crosses represent the local minima, the sites, connected by bonds through saddle points. The occupied bonds are represented by dark strokes, while the perturbed streamlines are represented by solid lines, schematically. The streamlines consist of the external and internal (darker solid lines enclosed by the occupied bonds) hull of the clusters of occupied bonds. 
It follows form (3.4) and (3.5) that the typical length of the external hull of the near critical level set is

$$
\ell(h) \sim h^{-v d_{H}}, \quad \text { for } \quad|h|<<\delta
$$

Moreover, since we assume that $|\nabla \psi|$ is bounded away from zero and infinity except at a set of discrete stagnation points, (3.4) gives the typical width of a web formed by the contours with the diameter of order $\xi$

$$
w(\xi) \sim \xi^{-1 / v}, \quad \text { for } \quad \xi>>\frac{1}{\delta^{v}}
$$

We will see in the next few sections that these power laws determine the asymptotic behavior of the effective diffusivity in the small molecular diffusion limit. Given the streamline geometry in the form of postulating the existence of trial functions that reflect this geometry (to be spelled out in Section 3.3 and 3.4), the main conclusion is as follows.

Given the exponents $\gamma$ and $d_{H}$ as in (3.3), (3.5), the effective diffusivity obeys the power law

$$
c_{1} \varepsilon^{\alpha} \leqslant \sigma_{\varepsilon} \leqslant c_{2} \varepsilon^{\alpha}, \quad \text { with } \quad \alpha=\frac{1}{2+v d_{H}} \in(0,1 / 2)
$$

where $v=\frac{4}{3}(\gamma+1) \in(0, \infty)$ and $c_{1}, c_{2}$ are constants as $\varepsilon \rightarrow 0$. In particular, when $\gamma=0$, and thus $v=\frac{4}{3}$ as in (3.4), the exponent $\alpha$ is

$$
\alpha=3 / 13
$$

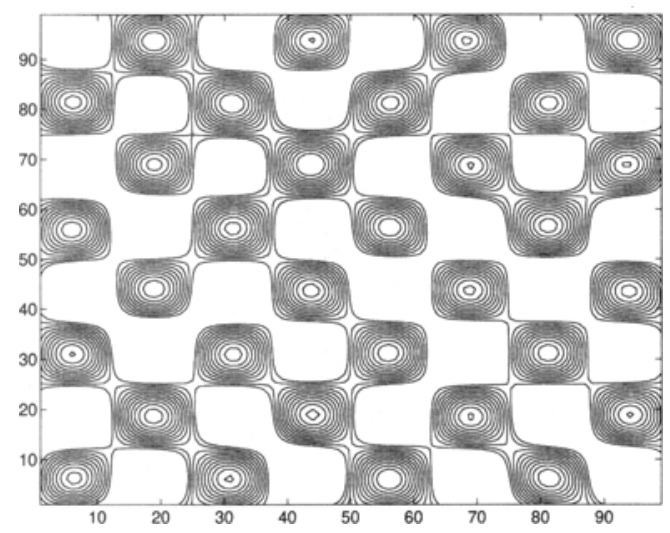

Fig. 5. Percolating level sets $h_{c}=0 \leqslant \psi \leqslant 1.0$. 
We will analyze the case $\gamma=0$ in the next few sections and then indicate how to generalize it at the end.

\subsection{Scaling Argument for the Effective Diffusivity}

We begin with a heuristic scaling argument based on the variational principles.

The effective diffusivity is the bulk transport coefficient influenced mostly by large scale excursions. In the case of the periodic cellular flow $\psi(x, y)=\sin x \cdot \sin y$, the diffusing Brownian particles travel long distances by hopping from one cell to another. They accomplish this by convection around the cells and by diffusion in a narrow strip near the separatrices (i.e. the boundary layer or the web). The width $w$ of the periodic boundary layer is determined by the balance of the diffusive flux across the layer and the convective flux along the layer in each cell. This balance is expressed by the equality of the diffusion time scale to the convection time scale

$$
\frac{w^{2}}{\varepsilon} \sim \frac{\ell}{u_{0}}
$$

where $\ell$ is the size of the cell and $u_{0}$ is the magnitude of the velocity, both of order 1 for $\psi(x \cdot y)=\sin x \cdot \sin y$. Thus

$$
w \sim \sqrt{\varepsilon}
$$

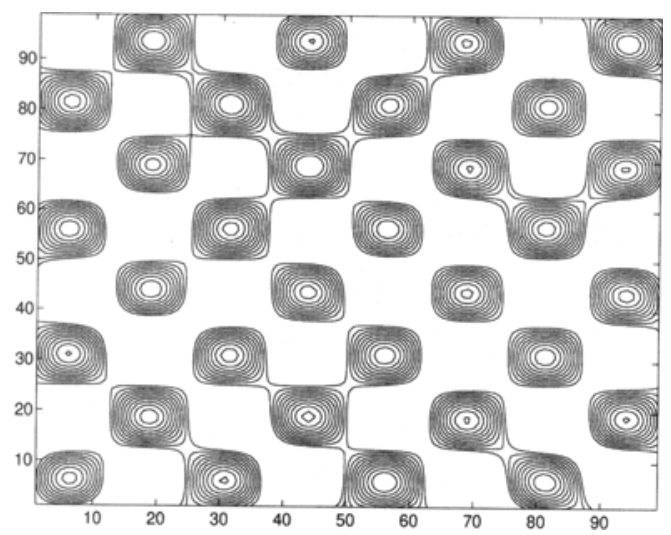

Fig. 6. Isolated islands of level sets $0.1 \leqslant \psi \leqslant 1.0$. 
The effective diffusivity $\sigma_{\varepsilon}$ can be obtained from (2.23). Roughly, the appropriate trial functions $f$ such that $\nabla f=\mathbf{F}$ should have large gradients of order $1 / \sqrt{\varepsilon}$ inside the boundary layer and be flat otherwise, while maintaining a unit average gradient. Thus the diffusive flux is

$$
\varepsilon \int_{[0,1]^{2}} d \mathbf{x} \mathbf{F} \cdot \mathbf{F} \sim \varepsilon\left(\frac{1}{\sqrt{\varepsilon}}\right)^{2} \cdot w \sim \sqrt{\varepsilon}
$$

and the convective flux is

$$
\frac{1}{\varepsilon} \int_{[0,1]^{2}} d \mathbf{x} \Gamma \tilde{\Psi}_{n} \mathbf{F} \cdot \Gamma \tilde{\Psi}_{n} \mathbf{F} \sim \sqrt{\varepsilon}
$$

since they are balanced by (3.10) and so $\sigma_{\varepsilon} \sim \sqrt{\varepsilon}$ for cellular flows. For the details of this calculation, we refer to ref. 9 .

We now apply the above scaling argument to the random stationary stream functions $\psi(x, y)$. The width of the fractal boundary layer associated with the critical level set $\psi(x, y)=h_{c}=0$ is determined by equating the diffusion time scale to the convection time scale. For $h>0$ this means that

$$
\frac{w^{2}(h)}{\varepsilon} \sim \frac{\ell(h)}{u_{0}}
$$

and as $h$ approaches $h_{c}=0, \ell(h)$ gets larger according to the power law (3.6), while the non-degeneracy of the stream function implies $u_{0}$ is of order one and $w(h) \sim h$. So (3.14) determines the typical level sets $\psi(x, y) \sim h_{\varepsilon}(\varepsilon)$ that contribute to large scale transport, as $\varepsilon \rightarrow 0$

$$
h_{\varepsilon} \sim \varepsilon^{1 /\left(2+v d_{H}\right)}
$$

With a web of this size, the diffusive flux will be

$$
\begin{aligned}
\varepsilon \int_{[0,1]^{2}} d \mathbf{x} \mathbf{F} \cdot \mathbf{F} & \sim \varepsilon\left(\frac{1}{h_{\varepsilon}}\right)^{2} \cdot \ell\left(h_{\varepsilon}\right) \cdot w\left(h_{\varepsilon}\right) \\
& \sim \varepsilon^{3 / 13}
\end{aligned}
$$

where $\ell\left(h_{\varepsilon}\right) \cdot w\left(h_{\varepsilon}\right)$ is the area of the boundary layer or web. The convective flux is expected to behave similarly because of the balance of time scales (3.14). Thus, the effective diffusivity behaves like

$$
\sigma_{\varepsilon} \sim c \varepsilon^{3 / 13}
$$


as $\varepsilon \rightarrow 0$, with $c$ a constant. In the analysis in the following sections we get instead of (3.17) the bounds

$$
c_{1} \varepsilon^{3 / 13} \leqslant \sigma_{\varepsilon} \leqslant c_{2} \varepsilon^{3 / 13}
$$

as $\varepsilon \rightarrow 0$, with constants $c_{1}$ and $c_{2}$. We use the variational principles (2.23) and (2.27), and the critical scaling laws (3.4), (3.5) and (3.6). $\nabla f$ is evidently aligned with the derivative with respect to $\bar{\psi}$ and hence $(3.20)$ immediately follows from the scaling of the stream variable.

\subsection{Upper Bound}

We first introduce the notion of boundary layer functions of scale $h$ for the periodized variational principles of section (2.3). For a periodized cell of size $n$ a boundary layer function of scale $h$ is one whose gradient is supported in the boundary layer $W_{h, n}=\left\{\left|\tilde{\psi}_{n}\right| \leqslant h\right\}$ and is asymptotically orthogonal to the velocity field, as $h \rightarrow 0$.

For the variational principles of section (2.3) we assume that there exist boundary layer trial functions $f_{h, n}$ of scale $h$ that satisfy the conditions

$$
\begin{gathered}
\varlimsup_{n \rightarrow \infty} \int_{[0,1]^{2}} d \mathbf{x}\left(\nabla f_{h, n}\right)^{2} \leqslant c \frac{\ell(h)}{h} \\
\varlimsup_{n, \int_{[0,1]^{2}}} d \mathbf{x}\left(\mathbf{u}_{n} \cdot \nabla f_{h, n}\right)^{2} \leqslant c u_{0}^{2} \frac{h}{\ell(h)}
\end{gathered}
$$

as $h \rightarrow 0$. To be admissible as a trial function for the periodized cell problems of sections 2.2 and $2.3, f_{h, n}$ must also satisfy the mean gradient, or the linear growth condition $(2.34,2.35)$. Here $h$ is a positive number that will be chosen to depend on $\varepsilon, h=h_{\varepsilon}$ and will tend to zero as $\varepsilon \rightarrow 0$ in a suitable way. When $h=h_{\varepsilon}$, we write $f_{h_{\varepsilon}, n}=f_{\varepsilon, n}$ and $\mathbf{F}_{h_{\varepsilon}, n}=\mathbf{F}_{\varepsilon, n}$ where $\mathbf{F}_{h, n}=\nabla f_{h, n}$. In the following, we denote a general constant independent of $\varepsilon$ and $n$ by $c$.

Let us explain why $(3.19,3.20)$ are compatible with our understanding of the percolation geometry near $h=0$. Since $f_{h, n}$ is flat everywhere except in the region between the external hull of $\left\{\tilde{\psi}_{n} \leqslant-h\right\}$ and $\left\{\tilde{\psi}_{n} \geqslant h\right\}$, the integrals in (3.19) and (3.20) are confined to the boundary layer region $W_{h, n}$. As explained in Section 3.1, both $\left\{\tilde{\psi}_{n} \leqslant-h\right\} \cap[0,1]^{2}$ and $\left\{\tilde{\psi}_{n} \geqslant h\right\}$ $\cap[0,1]^{2}$ consist approximately of $(n / \xi(h))^{2}$ connected components (clusters) with diameter $\xi_{n}(h)=\xi(h) / n$ and perimeter $\ell_{n}(h)=\ell(h) / n$, separated by $W_{h, n}$, in each of which the trial function $f_{h, n}$ is a constant. The change in $f_{h, n}$ across the boundary layer is chosen to be of order $\xi_{n}(h)$ 
so as to be consistent with the mean gradient condition (2.34). Therefore the cross-stream derivative of $f_{h, n}$ in $W_{h, n}$ is of order $\xi_{n}(h) / w_{n}(h)$ where $w_{n}(h)=w(h) / n$ is the width of $W_{h, n}$ and is proportional to $h / n$ for small $h$. In the direction parallel to the velocity, $f_{h, n}$ is chosen to vary in proportion to $\xi_{n}(h) / \ell_{n}(h)$ per unit length along $W_{h, n}$ so that the along-stream derivative of $f_{h, n}$ is of order $\xi_{n}(h) / \ell_{n}(h)$. This makes it consistent with the condition that the variation of $f_{h, n}$ from cluster to adjacent cluster be of order $\xi_{n}(h)$. Thus $\mathbf{F}_{h, n}$ points asymptotically for small $h$ in the cross-stream direction and is of order $\xi_{n}(h) / w_{n}(h)$. Condition (3.19) follows from these facts

$$
\begin{aligned}
\int_{[0,1]^{2}} d \mathbf{x} \mathbf{F}_{h, n} \cdot \mathbf{F}_{h, n} & \lessgtr c\left(\frac{\xi_{n}(h)}{w_{n}(h)}\right)^{2}\left(\frac{\xi(h)}{n}\right)^{-2} \ell_{n}(h) w_{n}(h) \\
& \lessgtr c \frac{h}{\ell(h)}
\end{aligned}
$$

Similarly, we have that

$$
\begin{aligned}
\int_{[0,1]^{2}} d \mathbf{x}\left(\mathbf{u}_{n} \cdot \nabla f_{h, n}\right)^{2} & \leqslant c u_{0}^{2}\left(\frac{\xi_{n}(h)}{\ell_{n}(h)}\right)^{2}\left(\frac{n}{\xi(h)}\right)^{2} \ell_{n}(h) w_{n}(h) \\
& \leqslant c u_{0}^{2} \frac{h}{\ell(h)}
\end{aligned}
$$

which is $(3.20)$.

The estimate for the diffusive term in (2.23) (the first term in the integral) is immediate from (3.19) or (3.21) and gives

$$
\varepsilon \int_{[0,1]^{2}} d \mathbf{x} \mathbf{F}_{h, n} \cdot \mathbf{F}_{h, n} \lesssim c \varepsilon \frac{h}{\ell(h)}
$$

The estimate for the convective flux (the second term in the integral in (2.23)) is more involved. If we write $\Gamma \Psi_{n} \mathbf{F}_{h, n}=\nabla f_{h, n}^{\prime}$, then $f_{h, n}^{\prime}$ is the $[-1,1]^{2}$-periodic solution of the rescaled Poisson equation

$$
\Delta f_{h, n}^{\prime}=n \mathbf{u}_{n} \cdot \nabla f_{h, n}
$$

and hence

$$
\frac{1}{\varepsilon} \int_{[0,1]^{2}} d \mathbf{x} \Gamma \tilde{\Psi}_{n} \mathbf{F}_{h, n} \cdot \Gamma \widetilde{\Psi}_{n} \mathbf{F}_{h, n}=\frac{1}{\varepsilon} \int_{[0,1]^{2}} d \mathbf{x} \nabla f_{h, n}^{\prime} \cdot \nabla f_{h, n}^{\prime}
$$


Now from the energy estimate for (3.24) we have that

$$
\begin{aligned}
\int_{[0,1]^{2}} d \mathbf{x} \nabla f_{h, n}^{\prime} \cdot \nabla f_{h, n}^{\prime} & \leqslant \sqrt{n^{2} \int_{W_{h, n}} d \mathbf{x}\left(\mathbf{u}_{n} \cdot \nabla f_{h, n}\right)^{2}} \cdot \sqrt{\int_{W_{h, n}} d \mathbf{x}\left(f^{\prime}\right)^{2}} \\
& \leqslant \sqrt{n^{2} \int_{W_{h, n}} d \mathbf{x}\left(\mathbf{u}_{n} \cdot \nabla f_{h, n}\right)^{2}} \cdot \sqrt{C_{n, n} \int_{W_{h, n}} d \mathbf{x}\left(\nabla f^{\prime}\right)^{2}}
\end{aligned}
$$

The constant $C_{h, n}$ is of order $(h / n)^{2}$, assuming $f_{h, n}^{\prime}$ is asymptotically a boundary layer function as $n \rightarrow \infty$. Thus we have for (3.25) the upper bound

$$
\frac{1}{\varepsilon} \int_{[0,1]^{2}} d \mathbf{x} \nabla f_{h, n}^{\prime} \cdot \nabla f_{h, n}^{\prime} \leqslant c u_{0}^{2} \frac{h^{3}}{\varepsilon \ell(h)}
$$

Adding (3.23) and (3.27) we have

$$
\begin{aligned}
& \varepsilon \int_{[0,1]^{2}} d \mathbf{x} \nabla f_{h, n} \cdot \nabla f_{h, n}+\frac{1}{\varepsilon} \int_{[0,1]^{2}} d \times \nabla f_{h, n}^{\prime} \cdot \nabla f_{h, n}^{\prime} \\
& \leqslant c \varepsilon \frac{\ell(h)}{h}+c u_{0}^{2} \frac{h^{3}}{\varepsilon \mathcal{\ell}(h)}
\end{aligned}
$$

which is an upper bound for the effective diffusivity by (2.23). This bound is optimal when the right side of (3.28) is minimized over $h$. The result is

$$
\varepsilon \frac{\ell\left(h_{\varepsilon}\right)}{h_{\varepsilon}} \sim u_{0}^{2} \frac{h_{\varepsilon}^{3}}{\varepsilon \ell\left(h_{\varepsilon}\right)}
$$

which determines the scale $h_{\varepsilon}$

$$
h_{\varepsilon} \sim \varepsilon^{3 / 13}
$$

The effective diffusivity is therefore bounded from above by

$$
\sigma_{\varepsilon} \leqslant c \varepsilon^{3 / 13}, \quad \text { as } \quad \varepsilon \rightarrow 0
$$

when (3.30) is substituted in (3.28).

Note that condition (3.29) is a precise version of the heuristic time balance (3.14) and is a consequence of the variational principle. 


\subsection{Lower Bound}

To get a lower bound for the effective diffusivity $\sigma_{\varepsilon}$, we use the dual variational principle (2.27). The trial functions $g_{h, n}$ will again be boundary layer functions of scale $h$ and they must satisfy the mean gradient condition (2.35).

The factor $1 /\left(\varepsilon^{2}+\widetilde{\psi}_{n}^{2}\right)$ in the integral in (2.27) and conditions $(3.19$, 3.20) suggest that we demand that the trial functions $g_{h, n}$ satisfy the conditions

$$
\begin{aligned}
\varlimsup_{n \rightarrow \infty} \int_{[0,1]^{2}} d \mathbf{x} \frac{1}{\tilde{\psi}_{n}^{2}} \nabla^{\perp} g_{h, n} \cdot \nabla^{\perp} g_{h, n} \leqslant c \frac{\ell(h)}{h^{3}} \\
\varlimsup_{n \rightarrow \infty} \int_{[0,1]^{2}} \frac{1}{\widetilde{\psi}_{n}^{2}}\left(\mathbf{u}_{n} \cdot \nabla g_{h, n}\right)^{2} \leqslant c u_{0}^{2} \frac{1}{\ell(h) h}
\end{aligned}
$$

where $u_{0}$ is a typical size for the velocity field and is of order one relative to $\varepsilon$. Since the integrals are mainly over the boundary layer region $W_{h, n}$, the streamfunction $\widetilde{\psi}_{n}$ is of order $h$ and hence conditions $(3.32,3.33)$ are the same as $(3.19,3.20)$ with the rightside divided by $h^{2}$. The existence of boundary layer functions $g_{h, n}$ that satisfy the conditions $(3.32,3.33)$ is therefore a consequence of the existence of boundary layer functions that satisfy conditions $(3.19,3.20)$.

Condition (3.32) gives immediately the upper bound on the diffusive resistance, the first term in the integral in (2.27),

$$
\varlimsup_{n \rightarrow \infty} \int_{[0,1]^{2}} d \mathbf{x} \frac{\varepsilon}{\varepsilon^{2}+\tilde{\psi}_{n}^{2}} \nabla^{\perp} g_{h, n} \cdot \nabla^{\perp} g_{h, n} \leqslant c \frac{\varepsilon \ell(h)}{h^{3}}
$$

The convective resistance, the second term in the integral in (2.27), is

$$
\frac{1}{\varepsilon} \int_{[0,1]^{2}} d \mathbf{x} \frac{1}{\varepsilon^{2}+\tilde{\psi}_{n}^{2}} \mathbf{G}_{h, n}^{\prime} \cdot \mathbf{G}_{h, n}^{\prime}
$$

with $\mathbf{G}_{h, n}^{\prime}=\nabla^{\perp} g_{h, n}^{\prime}$ and $g_{h, n}^{\prime}$ the $[-1,1]^{2}$-periodic solution of the dual Poisson equation

$$
\nabla^{\perp} \cdot \frac{1}{1+\frac{1}{\varepsilon^{2}} \tilde{\psi}_{n}^{2}} \nabla^{\perp} g_{h, n}^{\prime}=\nabla^{\perp} \cdot\left(\frac{\frac{1}{\varepsilon} \widetilde{\Psi}_{n}}{1+\frac{1}{\varepsilon^{2}} \widetilde{\psi}_{n}^{2}}\right) \nabla^{\perp} g_{h, n}=n \frac{1-\frac{1}{\varepsilon^{2}} \widetilde{\psi}_{n}^{2}}{\left(1+\frac{1}{\varepsilon^{2}} \widetilde{\psi}_{n}^{2}\right)^{2}} \mathbf{u}_{n} \cdot \nabla g_{h, n}
$$


This reduces to the following asymptotic equation as $\varepsilon \rightarrow 0$, after throwing away small terms

$$
\nabla^{\perp} \cdot \frac{1}{\tilde{\psi}_{n}^{2}} \nabla^{\perp} g_{h, n}^{\prime}=n \frac{\mathbf{u}_{n}}{\tilde{\psi}_{n}^{2}} \cdot \nabla g_{h, n}
$$

The energy estimate for (3.37) gives

$$
\begin{array}{rl}
\int_{[0,1]^{2}} & d \mathbf{x} \frac{1}{\tilde{\psi}_{n}^{2}} \nabla^{\perp} g_{h, n}^{\prime} \cdot \nabla^{\perp} g_{h, n}^{\prime} \\
& \leqslant c \sqrt{n^{2} \int_{W_{h, n}} d \mathbf{x} \frac{1}{\tilde{\psi}_{n}^{2}}\left(\mathbf{u}_{n} \cdot \nabla g_{h, n}\right)^{2}} \cdot \sqrt{C_{h, n} \int_{W_{h, n}} d \mathbf{x} \frac{1}{\tilde{\psi}_{n}^{2}} \nabla^{\perp} g_{h, n}^{\prime} \cdot \nabla^{\perp} g_{h, n}^{\prime}}
\end{array}
$$

Here the constant is again

$$
C_{h, n} \sim \frac{h^{2}}{n^{2}}
$$

assuming $g_{h, n}^{\prime}$ is a boundary layer function. The upper bound on the convective resistance follows immediately from (3.38) and (3.39)

$$
\frac{1}{\varepsilon} \int_{[0,1]^{2}} d \mathbf{x} \frac{1}{\varepsilon^{2}+\tilde{\psi}_{n}^{2}} \mathbf{G}_{h, n}^{\prime} \cdot \mathbf{G}_{h, n}^{\prime} \leqslant c u_{0}^{2} \frac{h}{\varepsilon \mathcal{\ell}(h)}
$$

Thus the effective resistivity $\sigma_{\varepsilon}^{-1}$ is bounded by

$$
\begin{aligned}
\sigma_{\varepsilon}^{-1} & \leqslant \varlimsup_{n \rightarrow \infty} \int_{[0,1]^{2}} d \mathbf{x} \frac{1}{\varepsilon^{2}+\tilde{\psi}_{n}^{2}}\left(\varepsilon\left|\nabla^{\perp} g_{h, n}\right|^{2}+\frac{1}{\varepsilon}\left|\nabla^{\perp} g_{h, n}^{\prime}\right|^{2}\right) \\
& \leqslant \frac{c \varepsilon \ell(h)}{h^{3}}+\frac{c u_{0}^{2} h}{\varepsilon \ell(h)}
\end{aligned}
$$

The optimal bound is achieved when

$$
\frac{\varepsilon \ell\left(h_{\varepsilon}\right)}{h_{\varepsilon}^{3}} \sim \frac{h_{\varepsilon} u_{0}^{2}}{\varepsilon \ell\left(h_{\varepsilon}\right)}
$$

that is,

$$
h_{\varepsilon} \sim \varepsilon^{3 / 13}
$$


Consequently,

$$
\sigma_{\varepsilon}^{-1} \leqslant c \varepsilon^{-3 / 13}
$$

The estimate (3.18) on the asymptotic behavior of $\sigma_{\varepsilon}$ then follows from the upper bound (3.31) and the lower bound (3.44).

\subsection{The Effective Diffusivity for General Cases}

For the more general case where $\gamma \in(-1, \infty)$ in (3.3), the exponent $v$ in (3.4) becomes

$$
v=\frac{4}{3}(\gamma+1) \in(0, \infty)
$$

The same analysis as before gives

$$
h_{\varepsilon} \sim \varepsilon^{1 /\left(2+v d_{H}\right)}
$$

with $d_{H}=7 / 4$, and the effective diffusivity is bounded by

$$
c_{1} \varepsilon^{\alpha} \leqslant \sigma_{\varepsilon} \leqslant c_{2} \varepsilon^{\alpha}, \quad \text { for } \quad \alpha=\frac{1}{2+v d_{H}}=\frac{3}{13+7 \gamma} \in(0,1 / 2)
$$

As $\gamma \rightarrow-1$, all the stagnation points tend to concentrate on the same level $\psi=0$ which is similar to the periodic cellular flow, and we have $\alpha \rightarrow 1 / 2$.

As $\gamma \rightarrow \infty$, the streamlines in the neighborhood of $\psi=0$ that contribute to the effective diffusivity have increasing perimeter. Since the effective diffusivity is an increasing function of the connected cluster we expect the exponent $\alpha$ in (3.47) to increase. In fact, $\alpha \rightarrow 0$ as $\gamma \rightarrow \infty$. This behavior is surprising and quite unlike the one for anisotropic open channels as in the cat's-eye flow (Fig. 2). The meandering isotropic streamlines have long but finite perimeters but there are no open channels that produce negative $\alpha$.

\section{RANDOM CELLULAR FLOWS IN CHECKERBOARD CONFIGURATIONS}

Let us consider the stream function $\sin x \sin y$ in the plane $R^{2}$. The separatrices, defined by $\sin x \sin y=0$, form a square lattice of size $\pi$. We call the streamline structure in each cell $[n \pi,(n+1) \pi] \times[k \pi,(k+1) \pi]$ an eddy and a cell with an eddy vortical. 
Let the eddy in each cell be suppressed, so that the velocity is zero in the cell, with probability $1-p, 0 \leqslant p \leqslant 1$. If this occurs, the cell is called vacant. In other words, a cell is vortical with probability $p$ and vacant with probability $1-p$, independently of the state of the remaining cells. The resulting stream function $\psi(x, y, \omega)$, where $\omega$ labels the configuration of vacant and vortical cells, is either $\sin x \sin y$ or 0 , depending on whether $(x, y)$ is in a vortical or a vacant cell. The infinite plane flow has effective diffusivity $\sigma_{\varepsilon}(\mathbf{e}, p)$ and we want to study the asymptotic behavior of $\sigma_{\varepsilon}(\mathbf{e}, p)$ as $\varepsilon \rightarrow 0$ for $0<p<1$.

There are two types of cell connections that are essential in characterizing the behavior of $\sigma_{\varepsilon}(\mathbf{e}, p)$ for small $\varepsilon$. We call two neighboring cells edge-connected if they share a common side and corner-connected if they share a common corner point, but not a common side. In a fixed square of size $2 n$, a horizontal (vertical) crossing is a chain of connected cells which connects $y=-n(X=-n)$ to $y=n(X=n)$. A crossing is edge-connected if neighboring cells in the chain are edge-connected. A crossing is cornerconnected if some neighboring cells in the chain are corner-connected. Following refs. 17 and 4, two edge-connected crossings are said to be disjoint if they do not overlap; two corner-connected crossings are said to be disjoint if they are separated by another crossing which does not overlap with them. In other words, two disjoint corner-connected crossings do not share any common side. They are, at best, corner-connected.

We know from percolation theory that as we vary $p$ we can distinguish three different regimes: $0 \leqslant p<1-p_{c}, 1-p_{c}<p<p_{c}, p_{c}<p \leqslant 1$, where $p_{c}=0.59 \ldots$ is the critical probability for site percolation in two-dimensions.

When $p_{c}<p \leqslant 1$ (Fig. 7) we have the strong percolation phase in which vortical cells form an infinite cluster in edge-connection and vacant cells

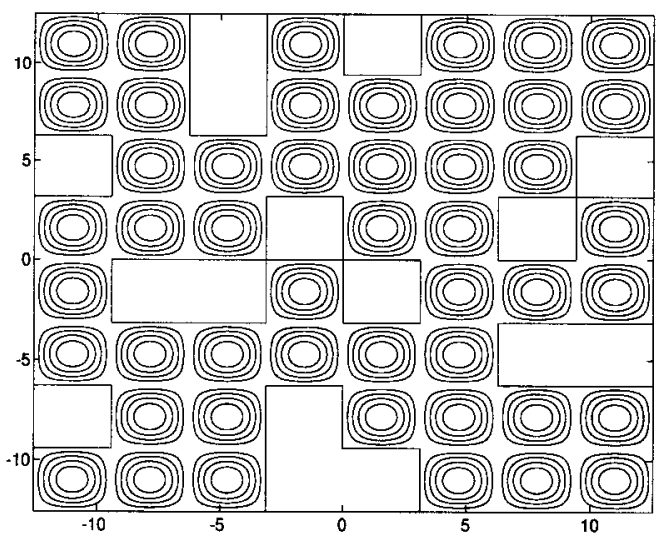

Fig. 7. Strong percolation phase: $p=0.7$. 
form isolated islands. More precisely, there exists a positive number $\alpha_{1}(p)$ such that

$$
\frac{1}{n} \#\left\{\begin{array}{c}
\text { disjoint horizontal (vertical) crossings of } \\
\text { edge connected vortical cells }
\end{array}\right\} \rightarrow \alpha_{1}(p)
$$

as $n \rightarrow \infty$, with probability one. Similarly, when $0 \leqslant p<1-p_{c}$ (Fig. 8) we have the non-percolation phase in which edge-connected vortical cells form isolated islands. We have that

$$
\frac{1}{n} \#\left\{\begin{array}{c}
\text { disjoint horizontal (vertical) crossings of } \\
\text { edge-connected vacant cells }
\end{array}\right\} \rightarrow \alpha_{2}(p)
$$

as $n \rightarrow \infty$, with probability one, for some positive $\alpha_{2}(p)$. Note that $\alpha_{2}(p)=\alpha_{1}(1-p)$.

When $1-p_{c}<p<p_{c}$ (Fig. 9), we have the weak percolation (or *-percolation) phase in which infinite, corner-connected clusters of vortical and vacant cells coexist and penetrate each other. More precisely,

$$
\begin{aligned}
& \frac{1}{n} \#\left\{\begin{array}{c}
\text { disjoint, corner-connected, horizontal (vertical) } \\
\text { crossings of vertical cells }
\end{array}\right\} \rightarrow \beta_{1}(p) \\
& \frac{1}{n} \#\left\{\begin{array}{c}
\text { disjoint, corner-connected, horizontal (vertical) } \\
\text { crossings of vacant cells }
\end{array}\right\} \rightarrow \beta_{2}(p)
\end{aligned}
$$

as $n \rightarrow \infty$, with probability one, for some positive $\beta_{1}(p)$ and $\beta_{2}(p)$. Note that $\beta_{2}(p)=\beta_{1}(1-p)$.

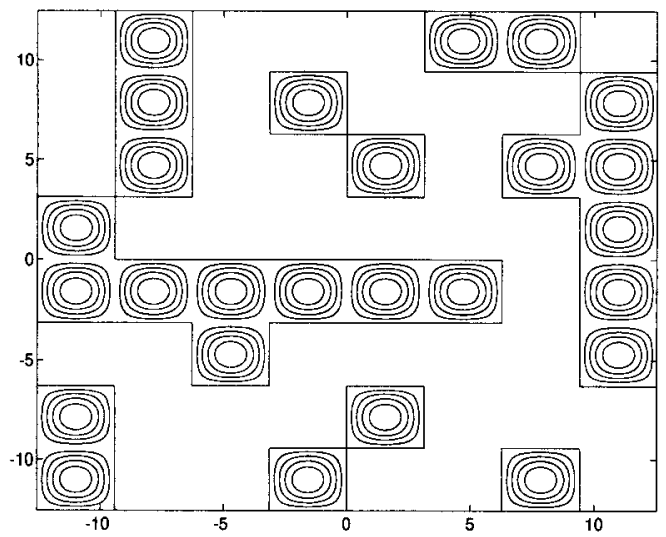

Fig. 8. Non-percolation phase: $p=0.3$. 


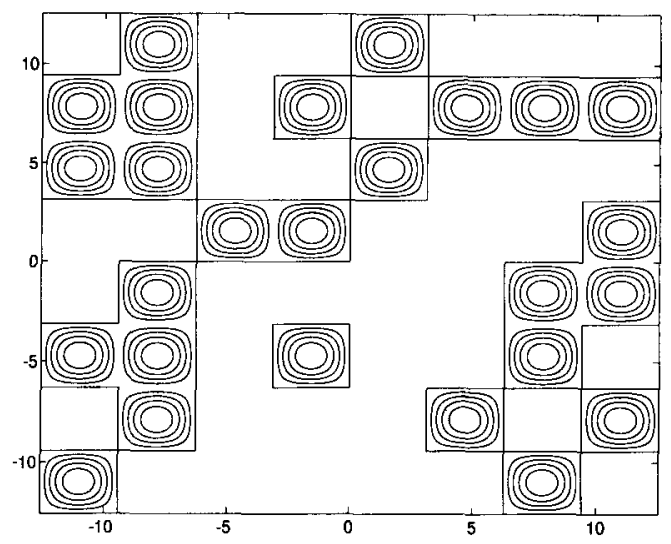

Fig. 9. Weak percolation phase: $p=0.5$.

Let us introduce a regularization of the flow around the corners, as in ref. 9. The flow is regularized so that the separatrices behave approximately like $s \sim \pm|t|^{1+\gamma}$, where $t$ and $s$ are tangential and normal coordinates, respectively and $\gamma$ measures the degree of contact of two separatrices near the contact point. With this regularization, the facts from percolation theory given above and the variational characterization for the effective diffusivity developed in the previous sections, we prove the following theorems.

Theorem 4.1. (i) When $p_{c}<p \leqslant 1$,

$$
c_{1} \sqrt{\varepsilon} \lesssim \sigma_{\varepsilon}(p) \lesssim c_{2} \sqrt{\varepsilon}, \quad \text { as } \quad \varepsilon \rightarrow 0
$$

for some constants $c_{1}, c_{2}>0$.

(ii) When $1-p_{c}<p<p_{c}$,

$$
\begin{array}{ll}
\sigma_{\varepsilon}(p) \sim c_{e}(p) \varepsilon^{1 / 2(1+1 /(1+2 \gamma))}, & \text { if } \gamma>0 \\
\sigma_{\varepsilon}(p) \sim c_{\varepsilon}(p) \varepsilon \log \frac{1}{\varepsilon}, & \text { if } \gamma=0
\end{array}
$$

where $c_{\varepsilon}(p)$ satisfies

$$
0<\liminf _{\varepsilon \rightarrow 0} c_{\varepsilon}(p) \leqslant \limsup _{\varepsilon \rightarrow 0} c_{\varepsilon}(p)<\infty
$$


and

$$
\lim _{\varepsilon \rightarrow 0} \frac{c_{\varepsilon}(p)}{c_{\varepsilon}\left(p^{\prime}\right)}=1, \quad \text { for all } \quad 1-p_{c}<p, \quad p^{\prime}<p_{c}
$$

(iii) When $0 \leqslant p<1-p_{c}$,

$$
\varepsilon \lesssim \sigma_{\varepsilon}(p) \lesssim c_{2} \varepsilon
$$

Here $\lesssim$ means $\leqslant$ to leading order, as $\varepsilon \rightarrow 0$, for some constants $c_{1}, c_{2}>0$.

Note that since the flow is isotropic, $\sigma_{\varepsilon}(p)$ is isotropic and thus a scalar. To fix ideas, we take $\mathbf{e}=(1,0)$ and estimate $\sigma_{\varepsilon}(\mathbf{e}, p)$ as $\varepsilon \rightarrow 0$. Before proving the theorem, we introduce an important result concerning the behavior of effective diffusivity as the probability $p$ varies.

Consider two step functions $\phi_{1}$ and $\phi_{2}$ that are either 1 or 0 in each cell $[\pi i, \pi(i+1)) \times[\pi j, \pi(j+1))$ and doubly periodic with a period $2 \pi n$. Let $\sigma_{k, \varepsilon}$ denote the effective diffusivities for the flows $\mathbf{u}_{k}=\phi_{k} \nabla^{\perp} \psi$, where $\psi=\sin x \sin y$. If $\phi_{2} \geqslant \phi_{1}$, then it is easy to see that

$$
\sigma_{2, \varepsilon}^{n} \gtrsim \sigma_{1, \varepsilon}^{n} \quad \text { as } \quad \varepsilon \rightarrow 0
$$

In other words, given the same streamline geometry, the stronger the flow the larger the enhancement of the diffusivity.

The following theorem is a probabilistic version of (4.11) which follows from (4.11) by adapting Kozlov's argument. ${ }^{(17)}$

Theorem 4.2. The effective diffusivity $\sigma_{\varepsilon}(p)$ is a non-decreasing function of $p$ as $\varepsilon \rightarrow 0$.

Proof. Let $\chi(x)$ be the characteristic function of the cell $[0, \pi] \times$ $[0, \pi]$. Then the stream function $\psi$ for the random cellular flow may be written as

$$
\psi(x, y)=\sum_{\mathbf{k} \in \mathbb{Z}^{2}} \sin x \sin y \xi_{\mathbf{k}} \chi(\mathbf{x}-\mathbf{k})
$$

where $\left\{\xi_{\mathbf{k}}\right\}_{\mathbf{k} \in \mathbb{Z}^{2}}$ are independent identically distributed random variables such that $P\left(\xi_{\mathbf{k}}=1\right)=p, P\left(\xi_{\mathbf{k}}=0\right)=1-p$. Consider a set of new i.i.d random variables $\left\{\xi_{\mathbf{k}}^{\prime}\right\}_{\mathbf{k} \in \mathbb{Z}^{2}}$ independent of $\left\{\xi_{\mathbf{k}}\right\}_{\mathbf{k} \in \mathbb{Z}^{2}}$ such that $P\left(\xi_{\mathbf{k}}^{\prime}=1\right)=$ $1-p^{\prime}, P\left(\xi_{\mathbf{k}}^{\prime}=0\right)=p^{\prime}$. Then the random variables $\zeta_{\mathbf{k}}=\xi_{\mathbf{k}} \xi_{\mathbf{k}}^{\prime}$ are independent and

$$
P\left(\zeta_{\mathbf{k}}=1\right)=p\left(1-p^{\prime}\right), \quad P\left(\zeta_{\mathbf{k}}=0\right)=1-p\left(1-p^{\prime}\right)
$$


Clearly, the flow with stream function

$$
\psi_{1}(x, y)=\sum_{\mathbf{k} \in \mathbb{Z}^{2}} \sin x \sin y \zeta_{\mathbf{k}} \chi(\mathbf{x}-\mathbf{k})
$$

has effective diffusivity $\sigma_{\varepsilon}\left(p\left(1-p^{\prime}\right)\right)$. Let $\varphi_{2}(x, y)=\sum_{\mathbf{k} \in \mathbb{Z}^{2}} \xi_{k} \chi(\mathbf{x}-\mathbf{k})$ and $\varphi_{1}(x, y)=\sum_{\mathbf{k} \in \mathbb{Z}^{2}} \zeta_{\mathbf{k}} \chi(\mathbf{x}-\mathbf{k})$. We have that

$$
\varphi_{2}(x, y)=\varphi_{1}(x, y)+\sum_{\mathbf{k} \in \mathbb{Z}^{2}} \xi_{\mathbf{k}}\left(1-\xi_{\mathbf{k}}^{\prime}\right) \chi(\mathbf{x}-\mathbf{k})
$$

since $\xi_{\mathbf{k}}=\xi_{\mathbf{k}} \xi_{\mathbf{k}}^{\prime}+\xi_{\mathbf{k}}\left(1-\xi_{\mathbf{k}}^{\prime}\right)=\xi_{\mathbf{k}}+\xi_{\mathbf{k}}\left(1-\xi_{\mathbf{k}}^{\prime}\right)$, and this implies $\varphi_{2}(x, y) \geqslant$ $\varphi_{1}(x, y)$. By $(4.11)$, we have that

$$
\sigma_{\varepsilon}\left(p\left(1-p^{\prime}\right)\right) \leqslant \sigma_{\varepsilon}(p) \quad \text { for } \quad \forall 0 \leqslant p, p^{\prime} \leqslant 1
$$

This completes the proof.

\subsection{Bounds for the Strong-Percolation Phase}

By the comparison theorem of the previous section, there is an obvious upper bound for $\sigma(p)$ independent of $p$, which is the effective diffusivity for the periodic cellular flow $\psi=\sin x \sin y$ calculated inref. 9 . A $p$-dependent upper bound can also be obtained as follows.

According to (4.1), there are $m \sim \alpha_{1}(p) n$, as $n \rightarrow \infty$, disjoint, vertical, edge-connected crossings of eddies. Let the trial function $f$ be flat between the crossings. Let it increase by $2 \pi n / m$ across each vertical crossing. Inside each crossing, $f$ is chosen to be of boundary layer type. We note that this trial function as constructed satisfies the flexible boundary condition

$$
f(n, y)-f(0, y)=n
$$

but not the rigid, linear Dirichlet condition nor the mixed DirichletNeumann condition of ref. 12 commonly used in the infinite volume limit for conductivity problems.

When this trial function is inserted into (2.23) it gives no contribution in the flat regions between the crossings and gives an order $(n / m)^{2} \sqrt{\varepsilon}$ contribution to the integral in each eddy of the crossings. The total number of eddies of the crossings is certainly less than $p \cdot n^{2}$ as $n \rightarrow \infty$. After summing over all edge-connected crossings, we have that

$$
\sigma_{\varepsilon}(p) \lesssim \frac{c \cdot p}{\alpha_{1}^{2}(p)} \sqrt{\varepsilon}
$$


where $c$ is the constant for the periodic cellular flow. ${ }^{(9)}$ It will be seen below that $(4.18)$ is a worse upper bound than $c \sqrt{\varepsilon}$, because $p / \alpha_{1}^{2}(p)$ will be shown to be greater than 1 .

For the lower bound, we make use of a set of $m \sim \alpha_{1}(p) n$, as $n \rightarrow \infty$ disjoint, horizontal, edge-connected crossings of eddies. The trial function $g$ is taken to be flat between the crossings and with a net decrease of $2 \pi n / m$ across each horizontal crossing. Note that $\nabla^{\perp} g=\mathbf{G}$ satisfies the mean field condition $\langle\mathbf{G}\rangle_{n}=\mathbf{e}=(1,0)$. In each cell of the crossings, $g$ is of boundary layer type. As before, using this $g$ in (2.27), we have the following inequality

$$
\sigma_{2}^{-1}(p) \lessgtr \frac{p}{\alpha_{1}^{2}(p)} \frac{1}{c \sqrt{\varepsilon}}
$$

or

$$
\frac{\alpha_{1}^{2}(p)}{p} c \sqrt{\varepsilon} \lesssim \sigma_{\varepsilon}(p) \quad \text { as } \quad \varepsilon \rightarrow 0
$$

where $c$ is the constant of the periodic cellular flow.

In view of (4.18) and (4.20), we conclude that $\alpha_{1}^{2}(p) \leqslant p$ and we have that

$$
\frac{\alpha_{1}^{2}(p)}{p} c \sqrt{\varepsilon} \lesssim \sigma_{\varepsilon}(p) \lesssim c \sqrt{\varepsilon}
$$

\subsection{Bounds for the Non-Percolation Phase}

We use boxes of size $n$ with order $n$ disjoint, edge-connected crossings of vacant cells to construct trial functions for upper as well as lower bounds. This is the dual of the strong-percolation phase. The trial functions are chosen to be flat between the crossings of vacant cells and to be linear across each crossing with slope $n / m$ where $m$ is the actual number of disjoint crossings in the $n$-box and $m \sim \alpha_{2}(p) n$, as $n \rightarrow \infty$. We note that this trial function satisfies the flexible boundary condition

$$
f(n, y)-f(0, y)=n
$$

but not the linear Dirichlet condition nor the mixed Dirichlet-Neumann conditions commonly used in conductivity problems. ${ }^{(12)}$ From this we get the bounds

$$
\varepsilon \lesssim \sigma_{\varepsilon}(p) \lesssim \frac{p}{\alpha_{2}^{2}(p)} \varepsilon
$$


The idea behind the lower bound in (4.21) for the strong percolation phase is to replace the flow between horizontal crossings by a vacant one. This corresponds to using trial flux functions that are flat between crossings. The resulting flow has a smaller effective diffusivity and is of order $\sqrt{\varepsilon}$ in the horizontal direction, as $\varepsilon \rightarrow 0$, because of the presence of order $n$ (as $n \rightarrow \infty$ ) horizontal, edge-connected crossings of vortical cells. To bound from above the effective diffusivity for the non-percolation phase, we replace the flow between vertical crossings by a vortical flow. This corresponds to using trial functions that are flat between crossings. The resulting flow has a bigger effective diffusivity, which is still of order $\varepsilon$ in the horizontal direction, as $\varepsilon \rightarrow 0$, because of the presence of order $n$ vertical, edge-connected crossings of vacant cells.

\subsection{Bounds for the Weak Percolation Phase}

The following analysis is based on an important observation made by Berlyand and Golden in ref. 4, and on the calculations for the periodic corner flows in ref. 9.

As noted before, in the intermediate weak percolation regime an infinite phase of vortical cells coexists with an infinite phase of vacant cells. The coexistence is made possible by corner connections. But not every corner connection is equally important. The special corner connections responsible for the enhancement of particle dispersion are the intersections of horizontal crossings of vortical cells and vertical crossings of vacant cells, or vice versa, which we call choke points. The collection of the vortical and vacant crossings associated with the set of choke points forms the backbone of the flow geometry. To avoid the singular nature of the corner flow we introduce a regularization as described in the beginning of this section.

Let $V_{n}$ be a maximal set of $m$ disjoint, vacant vertical crossings with $m$ of order $n$. On each crossing in $V_{n}$, a vacant cell connects to its neighboring cells either through an edge or a choke point. We enlarge each crossing in $V_{n}$ in a minimal way so that it also contains the vortical cells around its choke points. Because of the way disjointness of cornerconnected crossings is defined, the enlarged crossings still do not overlap with each other. Now we consider the following trial function $f(\mathbf{x})$. Between the enlarged crossings let $f(\mathbf{x})$ be flat, but let it increase by $\pi n / m$ across each enlarged crossing. The increase is linear across sections of the crossing with edge connections, and these portions of the function are continuously patched to the corner layer trial function near each choke point as done in ref. 9 for the periodic case. When this trial function is inserted 
in (2.23) it gives no contribution in the regions between the enlarged crossings, it gives $O\left((n / m)^{2} \varepsilon\right)$ on each crossing away from the choke points, and it gives $O\left((n / m)^{2} \varepsilon^{1 / 2(1+1 /(1+2 \gamma))}\right)$ at each choke point. After summing over all choke points, we get $\sigma_{\varepsilon}(p) \lesssim c_{2}(p) \varepsilon^{1 / 2(1+1 /(1+2 \gamma))}$, for some $c_{2}(p)>0$. Applying the dual variational principle (2.27) yields a corresponding lower bound $\sigma_{\varepsilon}(p) \gtrsim c_{1}(p) \varepsilon^{1 / 2(1+1 /(1+2 \gamma))}$, for some $c_{1}(p)>0$. This leads to

$\sigma_{\varepsilon}(p) \sim c_{\varepsilon}(p) \varepsilon^{1 / 2(1+1 /(1+2 \gamma))}, \quad 0<\liminf _{\varepsilon \rightarrow 0} c_{\varepsilon}(p) \leqslant \limsup _{\varepsilon \rightarrow 0} c_{\varepsilon}(p)<\infty$

if $\gamma>0$. When $\gamma=0$, we have

$$
\sigma_{\varepsilon}(p) \sim c_{\varepsilon}(p) \varepsilon \log \frac{1}{\varepsilon}, \quad 0<\liminf _{\varepsilon \rightarrow 0} c_{\varepsilon}(p), \quad \limsup _{\varepsilon \rightarrow 0} c_{\varepsilon}(p)<\infty
$$

The monotonicity of $\sigma_{\varepsilon}(p)$ in $p$ (Theorem 4.2) then implies that

$$
\liminf _{\varepsilon \rightarrow 0} \frac{c_{\varepsilon}(p)}{c_{\varepsilon}\left(p^{\prime}\right)} \leqslant 1, \quad \text { if } \quad 1-p_{c}<p<p^{\prime}<p_{c}
$$

It is clear from the variational analysis that only the choke points can contribute to the coefficient $c_{\varepsilon}(p)$ in (4.24) and (4.25). The contribution from all other structures is $o\left(\varepsilon^{1 / 2(1+1 /(1+2 \gamma))}\right)$. Since the choke structure and associated backbone are statistically invariant under the interchange of vacant and vortical cells, $c_{\varepsilon}(p)$ must be symmetric in the limit $\varepsilon \rightarrow 0$ and so

$$
\lim _{\varepsilon \rightarrow 0} \frac{c_{\varepsilon}(p)}{c_{\varepsilon}(1-p)}=1
$$

Thus, (4.26) and (4.27) establish the result (4.9) in Theorem 4.1.

The lack of a Keller-Dykhne type interchange identity, prevents us from showing the existence of $\lim _{\varepsilon \rightarrow 0} c_{\varepsilon}(p)$ and determining its exact value, as in ref. 4.

\section{APPENDIX A. CONVECTION ENHANCED DIFFUSION FOR PERIODIC CELLULAR FLOWS}

In this appendix we will show how to use the direct variational principles (2.23) and (2.25) to characterize the asymptotic behavior of the diffusion coefficient for the basic cellular flow with stream function $\psi(x, y)=\sin x \sin y$, when the diffusivity $\varepsilon$ goes to zero. This problem was analyzed in ref. 9 using the dual variational principles (2.23) and (2.27). 


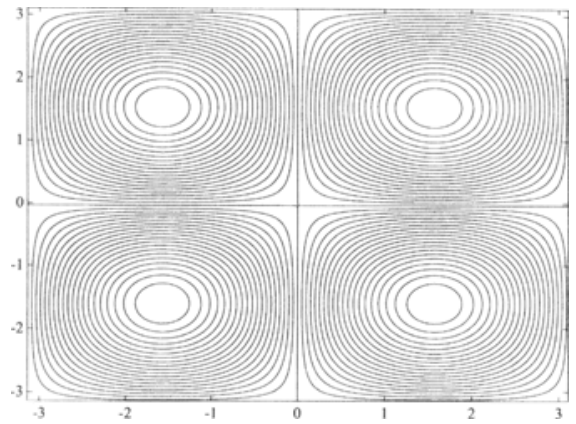

Fig. 10. The period cell.

The analysis given here illustrates another way to use the variational principles in what is perhaps the simplest, nontrivial convection diffusion problem.

The stream function $\psi(x, y)=\sin x \sin y$ gives rise to a cellular flow (Fig. 10). The cell problem for the effective diffusivity is (cf. ref. 9)

$$
\varepsilon \Delta \chi+\mathbf{u} \cdot \nabla \chi+\mathbf{u} \cdot \mathbf{e}=\mathbf{0}
$$

and determines, up to a constant, a periodic function $\chi(x, y),-\pi \leqslant x \leqslant \pi$, $-\pi \leqslant y \leqslant \pi$. The effective diffusivity is given by

$$
\sigma_{\varepsilon}(\mathbf{e})=\varepsilon\langle(\nabla \chi+\mathbf{e}) \cdot(\nabla \chi+\mathbf{e})\rangle
$$

where \langle\rangle is normalized integration over the period cell. When $\mathbf{e}=(1,0)$ is a unit vector in the $x$ direction then $\chi$ is odd in the $x$ direction and even in the $y$ direction. Problem (A.1) can then be restricted to a quarter of the cell, $0 \leqslant x \leqslant \pi, 0 \leqslant y \leqslant \pi$, and if we define

$$
\rho^{+}=\chi+x
$$

then

$$
\begin{gathered}
\varepsilon \Delta \rho^{+}+\mathbf{u} \cdot \nabla \rho^{+}=\mathbf{0} \\
\frac{\partial \rho^{+}}{\partial y}(x, 0)=\frac{\partial \rho^{+}}{\partial y}(x, \pi)=0 \\
\rho^{+}(0, y)=0, \quad \rho^{+}(\pi, y)=\pi
\end{gathered}
$$


and

$$
\sigma_{\varepsilon}(\mathbf{e})=\frac{\varepsilon}{\pi^{2}} \int_{0}^{\pi} \int_{0}^{\pi}\left[\left(\frac{\partial \rho^{+}}{\partial x}\right)^{2}+\left(\frac{\partial \rho^{+}}{\partial y}\right)^{2}\right] d x d y
$$

Now we introduce a new coordinate system $(x, y) \rightarrow(\psi, \theta)$ from the rectangle $0 \leqslant x \leqslant \pi, 0 \leqslant y \leqslant \pi$ to the region $\psi \geqslant 0,-4 \leqslant \theta \leqslant 4$ so that

$$
\nabla \psi \cdot \nabla \theta=0
$$

and

$$
|\nabla \theta|=|\nabla \psi|
$$

on the boundary of the rectangle. The circulation or angle variable $\theta(x, y)$ is determined by (A.8), (A.9), up to a constant. In general, it cannot be defined in all of the rectangle but only in a region including the boundary of the rectangle. We call the coordinates

$$
(h, \theta)=\left(\frac{\psi}{\sqrt{\varepsilon}}, \theta\right)
$$

the boundary layer coordinates. In terms of the boundary layer coordinates the cell problem (A.4)-(A.6) becomes

$$
|\nabla \psi|^{2} \frac{\partial^{2} \rho^{+}}{\partial h^{2}}+\sqrt{\varepsilon} \Delta \psi \frac{\partial \rho^{+}}{\partial h}+\varepsilon|\nabla \theta|^{2} \frac{\partial^{2} \rho^{+}}{\partial \theta^{2}}+\varepsilon \Delta \theta \frac{\partial \rho^{+}}{\partial \theta}+J \frac{\partial \rho^{+}}{\partial \theta}=0
$$

where $J=\psi_{y} \theta_{x}-\psi_{x} \theta_{y}=-\nabla^{\perp} \psi \cdot \nabla \theta$ is the Jacobian of the map $(x, y) \rightarrow$ $(\psi, \theta)$. Because of (A.9), $|\nabla \psi|^{2}=|J|$ at the boundary and hence the principal terms as $\varepsilon \rightarrow 0$ in (A.11) are

$$
\frac{\partial^{2} \rho^{+}}{\partial h^{2}}+\frac{\partial \rho^{+}}{\partial \theta}=0
$$

with

$$
\begin{aligned}
& \rho^{+}(0, \theta)=0, \quad 0<\theta<2 \\
& \frac{\partial \rho^{+}}{\partial h}(0, \theta)=0, \quad 2<\theta<4 \\
& \rho^{+}(0, \theta)=\pi, \quad-4<\theta<-2 \\
& \frac{\partial \rho^{+}}{\partial h}(0, \theta)=0, \quad-2<\theta<0
\end{aligned}
$$


From (A.7) we get that

$$
\sigma_{\varepsilon}(\mathbf{e}) \sim \sqrt{\varepsilon} \frac{1}{\pi^{2}} \int_{0}^{\infty} \int_{-4}^{4}\left(\frac{\partial \rho^{+}}{\partial h}\right)^{2} d h d \theta
$$

To make the above analysis precise we use the variational principles (2.23) and (2.25) as follows.

\section{A.1. Upper Bound for the Effective Diffusivity}

As in (A.12)-(A.13) we will fix $\mathbf{e}=\mathbf{e}_{1}=(1,0)$ since the case $\mathbf{e}=\mathbf{e}_{2}=(0,1)$ is similar. Let

$$
\begin{array}{r}
\mathscr{F}_{B L}=\left\{f=f(h, \theta), h \geqslant 0,-4 \leqslant \theta \leqslant 4, f \in C^{\infty},\right. \\
f \equiv \text { const for } h \geqslant N, \text { for some } N>0\}
\end{array}
$$

and suppose that $f \in \mathscr{F}_{B L}$ satisfies also the boundary conditions

$$
\begin{aligned}
f(0, \theta) & =0, & & 0<\theta<2 \\
\frac{\partial f}{\partial h}(0, \theta) & =0, & & -2<\theta<0, \quad 2<\theta<4 \\
f(0, \theta) & =\pi, & & -4<\theta<-2
\end{aligned}
$$

and the matching conditions on the separatrices

$$
\begin{aligned}
\int_{0}^{\infty} d h \frac{\partial f}{\partial \theta}=0, & -2<\theta<0, \quad 2<\theta<4 \\
\int_{0}^{\infty} d h \int_{\infty}^{h} \frac{\partial f}{\partial \theta}=0, & 0<\theta<2 \\
\int_{0}^{\infty} d h \int_{\infty}^{h} \frac{\partial f}{\partial \theta}=0, & -4<\theta<-2
\end{aligned}
$$

The matching conditions (A.17) are needed to ensure that the non local term in the functional satisfies the appropriate boundary conditions. This point will becomes clear in the following analysis. 
Consider now the direct variational principle (2.23), over the cell domain. We may look for trial fields $\mathbf{F}$ that have the quarter cell symmetry of (A.4)-(A.6). Then the averages can be restricted to a quarter cell also, and if $f \in \mathscr{F}_{B L}$ then $\mathbf{F}=\nabla f$ is an admissible trial field. We calculate $\nabla f$ and $\Gamma \Psi \nabla f$ for $f \in \mathscr{F}_{B L}$ and $\varepsilon$ small. We have that

$$
f_{x}=\frac{\psi_{x}}{\sqrt{\varepsilon}} \frac{\partial f}{\partial h}+\theta_{x} \frac{\partial f}{\partial \theta}, \quad f_{y}=\frac{\psi_{y}}{\sqrt{\varepsilon}} \frac{\partial f}{\partial h}+\theta_{y} \frac{\partial f}{\partial \theta}
$$

Then

$$
\begin{aligned}
\varepsilon\langle\mathbf{F} \cdot \mathbf{F}\rangle & \sim \frac{\varepsilon}{\pi^{2}} \int_{0}^{\infty} \int_{-4}^{4} \frac{1}{\varepsilon}|\nabla \psi|^{2} \frac{\sqrt{\varepsilon}}{J}\left(\frac{\partial f}{\partial h}\right)^{2} d h d \theta \\
& \sim \sqrt{\varepsilon} \frac{1}{\pi^{2}} \int_{0}^{\infty} \int_{-4}^{4}\left(\frac{\partial f}{\partial h}\right)^{2} d h d \theta
\end{aligned}
$$

since $|\nabla \psi|^{2} \sim|J|$ near $\psi=0$.

Similarly, let $(1 / \varepsilon) \Gamma \Psi \nabla f=\nabla f^{\prime}$ for some periodic $f^{\prime}$, then $f^{\prime}$ is the solution to the singular Poisson problem

$$
\varepsilon \Delta f^{\prime}=\mathbf{u} \cdot \nabla f
$$

and

$$
\frac{1}{\varepsilon}\langle\Gamma \Psi \nabla f \cdot \Gamma \Psi \nabla f\rangle=\varepsilon\left\langle\nabla f^{\prime} \cdot \nabla f^{\prime}\right\rangle
$$

As far as the energy integral $\varepsilon\left\langle\nabla f^{\prime} \cdot \nabla f^{\prime}\right\rangle$ is concerned, to leading order, it is sufficient to solve $f^{\prime}$ from the dominant terms in equation (A.20) after the boundary layer rescaling

$$
|\nabla \psi|^{2} \frac{\partial^{2}}{\partial h^{2}} f^{\prime} \sim J \frac{\partial}{\partial \theta} f
$$

which becomes

$$
\frac{\partial^{2}}{\partial h^{2}} f^{\prime} \sim \frac{\partial}{\partial \theta} f
$$

since $|\nabla \psi|^{2}=J$ on the separatrices. Equation (A.23) is an ordinary differential equation in $h$ and can be solved by direct integration. The matching 
conditions (A.17) guarantee that the solution $f^{\prime} \in \mathscr{F}_{B L}$ of (A.23) satisfies the boundary conditions

$$
\begin{gathered}
f^{\prime}(0, \theta)=0, \quad 0<\theta<2, \quad-4<\theta<-2 \\
\frac{\partial f^{\prime}}{\partial h}(0, \theta)=0, \quad-2<\theta<0, \quad 2<\theta<4
\end{gathered}
$$

From (A.20) we see that

$$
\begin{aligned}
\frac{1}{\varepsilon}\langle\Gamma \Psi \nabla f \cdot \Gamma \Psi \nabla f\rangle & \sim \sqrt{\varepsilon} \frac{1}{\pi^{2}} \int_{0}^{\infty} \int_{-4}^{4}\left(\int_{\infty}^{h} \frac{\partial f}{\partial \theta} d h^{\prime}\right)^{2} d h d \theta \\
& \sim \sqrt{\varepsilon} \frac{1}{\pi^{2}} \int_{0}^{\infty} \int_{-4}^{4}\left(\frac{\partial f^{\prime}}{\partial h}\right)^{2} d h d \theta
\end{aligned}
$$

Since $f \in \mathscr{F}_{B L}$ is identically zero for $h$ large the $h$ integrals are well defined. Using (A.19) and (A.25) in the variational principle (2.23) we have

$$
\sigma_{\varepsilon}(\mathbf{e}) \leqslant \varepsilon\langle\nabla f \cdot \nabla f\rangle+\frac{1}{\varepsilon}\langle\Gamma \Psi \nabla f \cdot \Gamma \Psi \nabla f\rangle
$$

and hence

$$
\varlimsup_{\varepsilon \downarrow 0} \frac{1}{\sqrt{\varepsilon}} \sigma_{\varepsilon}(\mathbf{e}) \leqslant \frac{1}{\pi^{2}} \int_{0}^{\infty} \int_{-4}^{4}\left\{\left(\frac{\partial f}{\partial h}\right)^{2}+\left(\int_{\infty}^{h} \frac{\partial f}{\partial \theta} d h^{\prime}\right)^{2}\right\} d h d \theta
$$

Since the left hand side does not depend on $f$ we also have

$$
\varlimsup_{\varepsilon \downarrow 0} \frac{1}{\sqrt{\varepsilon}} \sigma_{\varepsilon}(\mathbf{e}) \leqslant \inf _{\substack{f \in \mathscr{F}_{B L} \\ \text { (A.16), (A.17) }}} \frac{1}{\pi^{2}} \int_{0}^{\infty} \int_{-4}^{4}\left\{\left(\frac{\partial f}{\partial h}\right)^{2}+\left(\int_{\infty}^{h} \frac{\partial f}{\partial \theta} d h^{\prime}\right)^{2}\right\} d h d \theta
$$

\section{A.2. Lower Bound for the Effective Diffusivity}

Contrary to what we did in ref. 9, where we used the dual variational principle (2.27) to obtain the lower bound, here we get it from the direct maximum principle (2.25). The direct maximum principle is derived from the direct min-max principle (2.20) by elimination of the infimum. We have 


$$
\begin{aligned}
\sigma_{\varepsilon}(\mathbf{e}) & =\sup _{\substack{\nabla \times \mathbf{F}^{\prime}=0 \\
\left\langle\mathbf{F}^{\prime}\right\rangle=0}}\left\{\varepsilon\langle\mathbf{F} \cdot \mathbf{F}\rangle-2\left\langle\Psi \mathbf{F} \cdot \mathbf{F}^{\prime}\right\rangle-\varepsilon\left\langle\mathbf{F}^{\prime} \cdot \mathbf{F}^{\prime}\right\rangle\right\} \\
& =\sup _{\substack{\nabla \times \mathbf{F}^{\prime}=0 \\
\left\langle\mathbf{F}^{\prime}\right\rangle=0}}\left\{2\left\langle\Psi \mathbf{F}^{\prime}\right\rangle \cdot \mathbf{e}+\varepsilon|\mathbf{e}|^{2}-\varepsilon\left\langle\mathbf{F}^{\prime} \cdot \mathbf{F}^{\prime}\right\rangle-\frac{1}{\varepsilon}\left\langle\Gamma \Psi \mathbf{F}^{\prime} \cdot \Gamma \Psi \mathbf{F}^{\prime}\right\rangle\right\} \\
& \text { with } \nabla \cdot \mathbf{F}+\nabla \Psi \mathbf{F}^{\prime}=0, \quad\langle\mathbf{F}\rangle=\mathbf{e}
\end{aligned}
$$

Unlike the minimum principle, the maximum principle (A.29) is indefinite, and is therefore useful only when the nonlocal term $1 / \varepsilon\left\langle\Gamma \Psi^{\prime} \mathbf{F}^{\prime} \cdot \Gamma \Psi \mathbf{F}^{\prime}\right\rangle$ can be asymptotically evaluated in the limit $\varepsilon \rightarrow 0$, as in the case of periodic flows. However, for random flows, for which the boundary layer coordinates may not exit, it remains a technical difficulty. Instead of (A.29), we will actually use (A.28) because it preserves the symmetry between $\mathbf{F}$ and $\mathbf{F}^{\prime}$.

We consider the boundary layer trial function $f^{\prime} \in \mathscr{F}_{B L}$ such that the boundary conditions (A.24) and the following matching conditions are satisfied

$$
\begin{aligned}
\int_{0}^{\infty} d h \frac{\partial f^{\prime}}{\partial \theta} & =0, & & -2<\theta<0, \quad 2<\theta<4 \\
\int_{0}^{\infty} d h \int_{\infty}^{h} \frac{\partial f^{\prime}}{\partial \theta} & =\pi / 2, & & 0<\theta<2 \\
\int_{0}^{\infty} d h \int_{\infty}^{h} \frac{\partial f^{\prime}}{\partial \theta} & =-\pi / 2, & & -4<\theta<-2
\end{aligned}
$$

As in the analysis for the upper bound, we have that $\mathbf{F} \sim \nabla f$ where $f \in \mathscr{F}_{B L}$ and satisfies the boundary conditions (A.16) due to (A.31). We have

$$
\frac{\partial^{2}}{\partial h^{2}} f \sim \frac{\partial}{\partial \theta} f^{\prime}
$$

and

$$
\begin{aligned}
\langle\mathbf{F} \cdot \mathbf{F}\rangle & \sim \sqrt{\varepsilon} \frac{1}{\pi^{2}} \int_{0}^{\infty} \int_{-4}^{4}\left(\frac{\partial f}{\partial h}\right)^{2} d h d \theta \\
\left\langle\mathbf{F}^{\prime} \cdot \mathbf{F}^{\prime}\right\rangle & \sim \sqrt{\varepsilon} \frac{1}{\pi^{2}} \int_{0}^{\infty} \int_{-4}^{4}\left(\frac{\partial f^{\prime}}{\partial h}\right)^{2} d h d \theta \\
\left\langle\Psi \mathbf{F} \cdot \mathbf{F}^{\prime}\right\rangle & \sim \sqrt{\varepsilon} \frac{1}{\pi^{2}} \int_{0}^{\infty} \int_{-4}^{4}\left(h \frac{\partial f}{\partial h} \frac{\partial f^{\prime}}{\partial \theta}-h \frac{\partial f}{\partial \theta} \frac{\partial f^{\prime}}{\partial h}\right) d h d \theta
\end{aligned}
$$


With (A.33)-(A.35), (A.28) becomes

$$
\begin{aligned}
\liminf _{\varepsilon \rightarrow 0} \sigma_{\varepsilon}(\mathbf{e}) \leqslant & \sqrt{\varepsilon} \sup _{\substack{f^{\prime} \in \mathscr{F}_{B L} \\
(\mathrm{~A} .24),(\mathrm{A} .31)}} \frac{1}{\pi^{2}} \int_{0}^{\infty} \int_{-4}^{4} d h d \theta \\
& \times\left\{\left(\frac{\partial f}{\partial h}\right)^{2}-2 h\left(\frac{\partial f}{\partial h} \frac{\partial f^{\prime}}{\partial \theta}-\frac{\partial f}{\partial \theta} \frac{\partial f^{\prime}}{\partial h}\right)-\left(\frac{\partial f^{\prime}}{\partial h}\right)^{2}\right\}
\end{aligned}
$$

\section{A.3. Equality of Upper and Lower Bounds}

We now show that the upper bound (A.27) is equal to the the lower bound (A.36) and that they coincide with the constant in (A.14), obtained by solving (A.12)-(A.13). This will prove

Theorem A.1. The limit

$$
\lim _{\varepsilon \downarrow 0} \frac{1}{\sqrt{\varepsilon}} \sigma_{\varepsilon}(\mathbf{e})=\frac{1}{\pi^{2}} \int_{0}^{\infty} \int_{-4}^{4}\left(\frac{\partial \rho^{+}}{\partial h}\right)^{2} d h d \theta
$$

exists and equals the right side.

Proof. We begin with (A.12) and write it in divergence form

$$
\partial \cdot\left(I_{1} \pm \mathbf{h}\right) \partial \rho^{ \pm}=\mathbf{0}
$$

where

$$
\begin{gathered}
\partial=\left(\frac{\partial}{\partial h}, \frac{\partial}{\partial \theta}\right) \\
I_{1}=\left(\begin{array}{ll}
1 & 0 \\
0 & 0
\end{array}\right), \quad \mathbf{h}=\left(\begin{array}{cc}
0 & h \\
-h & 0
\end{array}\right)
\end{gathered}
$$

Both $\rho^{+}$and $\rho^{-}$are to satisfy the boundary conditions (A.13). We define

$$
c^{*}(\mathbf{e})=\frac{1}{\pi^{2}} \int_{0}^{\infty} \int_{-4}^{4}\left(\frac{\partial \rho^{+}}{\partial h}\right)^{2} d h d \theta
$$

We proceed now to symmetrize this problem. Let

$$
\rho=\frac{\rho^{+}+\rho^{-}}{2}, \quad \rho^{\prime}=\frac{\rho^{+}-\rho^{-}}{2}
$$


Then $\rho$ and $\rho^{\prime}$ satisfy

$$
\frac{\partial^{2} \rho}{\partial h^{2}}+\frac{\partial \rho^{\prime}}{\partial \theta}=0, \quad \frac{\partial^{2} \rho^{\prime}}{\partial h^{2}}+\frac{\partial \rho}{\partial \theta}=0
$$

in terms of which $c^{*}(\mathbf{e})$ admits a min-max variational formulation

$$
\begin{aligned}
c^{*}(\mathbf{e})= & \frac{1}{\pi^{2}} \int_{0}^{\infty} \int_{-4}^{4} d h d \theta\left\{\left(\frac{\partial \rho}{\partial h}\right)^{2}+\left(\frac{\partial \rho^{\prime}}{\partial h}\right)^{2}\right\} \\
= & \frac{1}{\pi^{2}} \int_{0}^{\infty} \int_{-4}^{4} d h d \theta\left(\begin{array}{cc}
-I_{1} & -\mathbf{h} \\
\mathbf{h} & I_{1}
\end{array}\right)\left(\begin{array}{c}
\partial \rho^{\prime} \\
\partial \rho
\end{array}\right) \cdot\left(\begin{array}{c}
\partial \rho^{\prime} \\
\partial \rho
\end{array}\right) \\
= & \frac{1}{\pi^{2}} \int_{0}^{\infty} \int_{-4}^{4} d h d \theta\left\{\left(\frac{\partial \rho}{\partial h}\right)^{2}-2 h\left(\frac{\partial \rho}{\partial h} \frac{\partial \rho^{\prime}}{\partial \theta}-\frac{\partial \rho}{\partial \theta} \frac{\partial \rho^{\prime}}{\partial h}\right)-\left(\frac{\partial \rho^{\prime}}{\partial h}\right)^{2}\right\} \\
= & \inf _{\substack{f \in \mathscr{F}_{B L} \\
(\mathrm{~A} .16),(\mathrm{A} .17)}} \sup _{\substack{f^{\prime} \in \mathscr{F}_{B L} \\
(\mathrm{~A} .24),(\mathrm{A} .31)}} \frac{1}{\pi^{2}} \int_{0}^{\infty} \int_{-4}^{4} d h d \theta \\
& \times\left\{\left(\frac{\partial f}{\partial h}\right)^{2}-2 h\left(\frac{\partial f}{\partial h} \frac{\partial f^{\prime}}{\partial \theta}-\frac{\partial f}{\partial \theta} \frac{\partial f^{\prime}}{\partial h}\right)-\left(\frac{\partial f^{\prime}}{\partial h}\right)^{2}\right\}
\end{aligned}
$$

By eliminating the supremum and infimum, we obtain the minimum principle and maximum principle respectively

$$
\begin{aligned}
& c^{*}(\mathbf{e})= \inf _{\substack{f \in \mathscr{F}_{B L} \\
(\mathrm{~A} .16)_{(\mathrm{A}) .17)}}} \frac{1}{\pi^{2}} \int_{0}^{\infty} \int_{-4}^{4}\left\{\left(\frac{\partial f}{\partial h}\right)^{2}+\left(\int_{\infty}^{h} \frac{\partial f}{\partial \theta} d h^{\prime}\right)^{2}\right\} d h d \theta \\
& c^{*}(\mathbf{e})= \sup _{\substack{f^{\prime} \in \mathscr{F}_{B L} \\
(\mathrm{~A} .24),(\mathrm{A} .31)}} \frac{1}{\pi^{2}} \int_{0}^{\infty} \int_{-4}^{4} d h d \theta \\
& \times\left\{\left(\frac{\partial f}{\partial h}\right)^{2}-2 h\left(\frac{\partial f}{\partial h} \frac{\partial f^{\prime}}{\partial \theta}-\frac{\partial f}{\partial \theta} \frac{\partial f^{\prime}}{\partial h}\right)-\left(\frac{\partial f^{\prime}}{\partial h}\right)^{2}\right\} \\
& \text { with } \frac{\partial^{2}}{\partial h^{2}} f+\frac{\partial}{\partial \theta} f^{\prime}=0
\end{aligned}
$$

This shows the desired equality. 


\section{ACKNOWLEDGMENTS} models.

We thank Jennifer Chayes for a helpful dscussion of percolation

This work was mostly done while A.F. was visiting at the Department of Mathematics, UCLA, and was supported by DARPA grant URI N00014092-J-1890 and NSF grant DMS-9306720. The work of G.P. was supported by NSF grant DMS 9308471 and AFSOR grant F49620-94-10436.

\section{REFERENCES}

1. K. S. Alexander and S. A. Molchanov, "Percolation of Level Sets for Two-dimensional Random Fields with Lattice Symmetry," J. Stat. Phys. 77:627-643, no. 3-4 (1994).

2. M. Avellaneda and A. J. Majda, "An Integral Representation and Bounds on the Effective Diffusivity in Passive Advection by Laminar and Turbulent Flows," Commun. Math. Phys. 138:339-391 (1991).

3. A. Bensoussan, J. L. Lions and G. C. Papanicolaou, "Asymptotic Analysis for Periodic Structures," North-Holland, Amsterdam (1978).

4. L. Berlyand and K. Golden, "Exact Results for the Effective Conductivity of a Continuum Percolation Model," Phys. Rev. B 50:2114-2117 (1994).

5. A. V. Cherkaev and L. V. Gibiansky, "Variational Principles for Complex Conductivity, Viscoelasticity and Similar Problems in Media with Complex Moduli," J. Math. Phys. 35:1, 127-145 (1994).

6. S. Childress, "Alpha-Effect in Flux Ropes and Sheepts," Phys. Earth Planet Inter. 20:172-180 (1979).

7. S. Childress and A. M. Soward, "Scalar Transport and Alpha-Effect for a Family of Cat's-Eye Flows," J. Fluid Mech. 205:99-133 (1989).

8. J. L. Doob, "Stochastic Processes," John Wiley \& Sons, Inc. 1953.

9. A. Fannjiang and G. C. Papanicolaou, "Convection Enhanced Diffusion for Periodic Flows," SIAM J. Appl. Math. 54:2, 333-408 (1994).

10. A. Fannjiang and G. C. Papanicolaou, "Diffusion in Turbulence," Prob. Theory and Rel. Fields 105:279-334 (1996).

11. A. Fannjiang and T. Komorowski, "An Martingale Approach to Homogenization of Unbounded Random Flows," to appear in Annals of Probability.

12. K. Golden and G. C. Papanicolaou, "Bounds for Effective Parameters of Heterogeneous Media by Analytic Continuation," Commun. Math. Phys. 90:473-491 (1983).

13. M. B. Isichenko, "Percolation, Statistical Topography, and Transport in Random Media," Rev. Mod. Phys. 64:4, 961-1043 (1992).

14. A. V. Gruznov, M. B. Isichenko, and J. Kalda, "Two-dimensional Turbulent Diffusion," Sov. Phys. JETP 70:263-269 (1990).

15. M. B. Isichenko and J. Kalda, "Statistical Topography II. 2-D Transport of a Passive Scalar," J. Nonlinear Sci. 1:375-396 (1991).

16. S. M. Kozlov, "The Method of Averaging and Walks in Inhomogeneous Environments," Russian Math. Surveys 40:2, 73-145 (1985).

17. S. M. Kozlov, "Geometric Aspects of Averaging," Russian Math. Surveys 44:2, 91-144 (1989). 
18. M. P. M. den Nijs, "A Relation Between the Temperature Exponents of the Eight-Vertex and $q$-State Potts Model," J. Phys. A12:1857 (1979).

19. G. C. Papanicolaou and S. Varadhan, "Boundary Value Problems with Rapidly Oscillating Random Coefficients," Colloquia Mathematica Sicietatis Janos Bolyai 27, Random Fields, Esztergom (Hungary) 1979, Amsterdam, North-Holland, 1982, pp. 835-873.

20. M. N. Rosenbluth, H. L. Berk, I. Doxas, and W. Horton, "Effective Diffusion in Laminar Convective Flows," Phys. Fluids 30:2636-2647 (1987).

21. B. Shraiman, "Diffusive Transport in a Rayleigh-Benard Convection Cell," Phys. Rev. A 36:261 (1987).

22. H. Saleur and B. Duplantier, "Exact Determination of the Percolation Hull Exponent in Two Dimension," Phys. Rev. Lett. 51:2048 (1985).

23. A. Weinrib, "Percolation Threshold of a Two-Dimensional Continuum System," Phys. Rev. B 26:1352 (1982).

24. R. M. Ziff, "Test of Scaling Exponents for Percolation-Cluster Perimeters," Phys. Rev. Lett. 56:545 (1986).

25. J. M. Ziman, "Models of Disorder," Cambridge University, New York (1979). 